ARTICLE

DOI: $10.1038 / s 41467-018-03050-0$

OPEN

\title{
Vascular niche IL-6 induces alternative macrophage activation in glioblastoma through HIF-2 $\alpha$
}

Qirui Wang ${ }^{1,2}$, Zhenqiang He ${ }^{1,3}$, Menggui Huang ${ }^{1}$, Tianrun Liu¹,4, Yanling Wang ${ }^{1}$, Haineng Xu1, Hao Duan ${ }^{1,3}$, Peihong Ma', Lin Zhang (10) ${ }^{5}$, Scott S. Zamvil ${ }^{6}$, Juan Hidalgo (1) ${ }^{7}$, Zhenfeng Zhang (1) ${ }^{8}$, Donald M. O'Rourke ${ }^{9}$, Nadia Dahmane ${ }^{9}$, Steven Brem ${ }^{9}$, Yonggao Mou ${ }^{3}$, Yanqing Gong ${ }^{10}$ \& Yi Fan ${ }^{1,9}$

Spatiotemporal regulation of tumor immunity remains largely unexplored. Here we identify a vascular niche that controls alternative macrophage activation in glioblastoma (GBM). We show that tumor-promoting macrophages are spatially proximate to GBM-associated endothelial cells (ECs), permissive for angiocrine-induced macrophage polarization. We identify ECs as one of the major sources for interleukin-6 (IL-6) expression in GBM microenvironment. Furthermore, we reveal that colony-stimulating factor- 1 and angiocrine IL-6 induce robust arginase- 1 expression and macrophage alternative activation, mediated through peroxisome proliferator-activated receptor- $\gamma$-dependent transcriptional activation of hypoxiainducible factor- $2 \alpha$. Finally, utilizing a genetic murine GBM model, we show that EC-specific knockout of IL-6 inhibits macrophage alternative activation and improves survival in the GBM-bearing mice. These findings illustrate a vascular niche-dependent mechanism for alternative macrophage activation and cancer progression, and suggest that targeting endothelial IL-6 may offer a selective and efficient therapeutic strategy for GBM, and possibly other solid malignant tumors.

\footnotetext{
${ }^{1}$ Department of Radiation Oncology, University of Pennsylvania Perelman School of Medicine, Philadelphia, PA 19104, USA. ${ }^{2}$ School of Traditional Chinese Medicine, Southern Medical University, 510515 Guangzhou, China. ${ }^{3}$ Department of Neurosurgery, Sun Yat-sen University Cancer Center, 510060 Guangzhou, China. ${ }^{4}$ Department of Otorhinolaryngology, Division of Head and Neck Surgery, The Sixth Affiliated Hospital of Sun Yat-sen University, 510655 Guangzhou, China. ${ }^{5}$ Department of Obstetrics and Gynecology, University of Pennsylvania Perelman School of Medicine, Philadelphia, PA 19104, USA. ${ }^{6}$ Department of Neurology and Program in Immunology, University of California at San Francisco, San Francisco, CA 94158, USA. ${ }^{7}$ Department of Cellular Biology, Physiology, and Immunology, Autonomous University of Barcelona, 08193 Barcelona, Spain. ${ }^{8}$ Department of Radiology The, Second Affiliated Hospital of Guangzhou Medical University, 510260 Guangzhou, China. ${ }^{9}$ Department of Neurosurgery, University of Pennsylvania Perelman School of Medicine, Philadelphia, PA 19104, USA. ${ }^{10}$ Department of Medicine, Division of Human Genetics and Translational Medicine, University of Pennsylvania Perelman School of Medicine, Philadelphia, PA 19104, USA. Correspondence and requests for materials should be addressed to Y.F. (email: fanyi@uphs.upenn.edu)
} 
$\mathrm{M}$ ost malignant solid tumors are characterized by extensive infiltration of inflammatory leukocytes. Among them, tumor-associated macrophages play a pivotal role in tumor growth, cancer immunosuppression, and therapy resistance ${ }^{1-3}$. In contrast to classically activated macrophages that stimulate phagocytosis, inflammation, and host immunity, a prominent population of macrophages in tumor microenvironment undergoes alternative activation to acquire tumor-promoting functions, for example, these macrophages express anti-inflammatory cytokines, such as interleukin-10 (IL10 ), and tumor growth factor- $\beta$ (TGF- $\beta$ ), and arginase- 1 that inhibits nitric oxide (NO) production and produces ornithine ${ }^{4-7}$. Growing evidence suggests that alternative macrophage activation is a driving force that fuels cancer progression, but the underlying tumor microenvironment-dependent mechanisms remain largely unknown.

Glioblastoma multiforme (GBM), the grade IV glioma, is the most common and most aggressive primary brain tumor. GBM is among the most lethal of human malignancies, with a current median overall survival of approximately 14 months ${ }^{8,} 9$, largely due to its high resistance to standard-of-care treatments including surgical resection, radiation, and chemotherapy ${ }^{10}$. The development of new therapies is therefore urgently needed, in which targeting tumor immunity holds great promise for GBM treatment. Notably, macrophages are a major population of the nonneoplastic cells in GBM, evidenced by as many as half of the cells in GBM tumors are macrophages or microglia ${ }^{11,12}$, suggesting that tumor-associated macrophages may represent an indispensable target for immunotherapy. Likewise, a recent study shows that receptor inhibition of colony-stimulating factor-1 (CSF-1), a major factor for macrophage differentiation and survival, alters alternative macrophage polarization and blocks GBM progression $^{13}$.

A multitude of evidence shows that macrophages stimulate glioma growth and invasion and induce therapeutic resistance $^{12,14}$. Glioma-associated macrophages express and secrete multiple factors including STI1, EGF (epidermal growth factor), TGF- $\beta$, and MT1-MMP to promote glioma cell survival, proliferation, and migration ${ }^{15-19}$. On the other hand, glioma cells induce macrophage recruitment by releasing chemoattractants CXCL12, GDNF, and CSF-1 ${ }^{19-21}$. However, how macrophage activation is spatiotemporally regulated in glioma is largely unclear, which is critical for the development of new therapies against GBM. Here, we reveal a vascular niche-dependent regulatory system for macrophage activation, targeting which may offer new therapeutic opportunities for the treatment of GBM, and possibly other solid malignant tumors.

\section{Results}

Vasculature-associated alternative macrophage activation. We investigated potential alternative macrophage activation in human GBM tumors. Although there are currently no specific surface markers identified for distinct macrophage activation, alternatively activated macrophages reliably express CD206 and CD163 (and anti-inflammatory cytokine IL-10), in contrast to the expression of CD86 (and proinflammatory cytokine IL-12) by classically activated macrophages ${ }^{4,22}$. Immunofluorescence analysis of surgical tumor specimens from human patients with different grades of gliomas showed that a large population of GBM-associated $\mathrm{CD}^{+} 8^{+}$macrophages robustly expressed CD206 and CD163 (Fig. 1a, b) and relatively expressed CD86 at a lower level (Supplementary Fig. 1), while only small population of $\mathrm{CD}^{+}{ }^{+}$macrophages or microglia cells expressed CD206 in normal brains (Supplementary Fig. 1). Moreover, consistent with previously published work showing that glioma grades correlate with the expression of multiple alternative activation markers in tumor-associated macrophages ${ }^{23}$, there was an increase in CD206 expression by tumor-associated macrophages from different grades of gliomas (Fig. 1c), suggesting enhanced alternative activation in these macrophages. As a critical marker for the antiinflammatory macrophage subset, arginase-1 competes with inducible nitric oxide synthase (iNOS) and hydrolyzes L-arginine into urea and ornithine, a precursor to L-proline and polyamines, which suppress NO-mediated cytotoxicity via L-arginine consumption, enhance collagen synthesis and fibrosis via L-ornithine formation, and increase cellular proliferation via polyamine generation, all important for macrophage-mediated tumor-promoting functions ${ }^{24,25}$. Our data indicate that a majority of GBMassociated macrophages expressed arginase-1 (Supplementary Fig. 2), verifying the increased alternative activation of macrophages in GBM.

Interestingly, immunofluorescence analysis of these human specimens indicated that $\mathrm{CD} 206^{+} \mathrm{CD} 68^{+}$macrophages were localized proximately to $\mathrm{CD} 31^{+}$vascular endothelial cells (ECs) (Fig. 1d). To spatially precisely analyze their distribution, we took advantage of a fluorescence protein-based genetic labeling system to visualize the vasculature, in which tdTomato expression is driven by EC-specific Tie2 promoter in mice (Fig. 1e). An orthotopic, genetic murine GBM model with a native microenvironment was induced by $R C A S / N$-tva-mediated somatic PDGF gene transfer in Ink4a-Arf ${ }^{-/} ; \mathrm{Pten}^{-/-}$neural stem/ progenitor cells, followed by tumor transplantation into the Tie2-Cre;ROSA-LSL-tdTomato mice (Fig. 1f). Consistent with the observations in human subjects, three-dimensional reconvolution of confocal scanning images showed that $\mathrm{F} 4 / 80^{+} \mathrm{CD} 206^{+}$macrophages were localized near the tdTomato $^{+}$ECs (Fig. If and Supplementary Movie 1), implicating a possible role of tumorassociated ECs in alternative macrophage activation in GBM.

Glioma ECs promote alternative macrophage polarization. To test the role of tumor-associated ECs in macrophage alternative activation, we pretreated mouse ECs with the conditioned medium (CM) harvested from the medium supernatant of cultured mouse GL26 glioma cells, and co-cultured these ECs with mouse bone marrow (BM) cells. CD11 ${ }^{+}$macrophages were analyzed by flow cytometry for macrophage activation. Our data revealed that co-culture with control ECs, and to a greater extent, with gliomaCM-pretreated ECs, induced robust CD206 expression: notably, over $60 \%$ of $\mathrm{CD}_{11 \mathrm{~b}^{+}}$macrophages positively expressed CD206 when co-cultured with glioma-CM-pretreated ECs, implicating that glioma microenvironment-stimulated ECs promote alternative activation of macrophage (Fig. 2a). In contrast, both CSF-1 treatment and co-culture with ECs induced comparable CD86 expression in $\mathrm{CD}_{11} \mathrm{~b}^{+}$macrophages. Additionally, the robust CD206 expression in macrophages induced by co-cultured glioma-CM-pretreated ECs was validated by immunofluorescence (Supplementary Fig. 3). To specifically analyze classically and alternatively activated macrophages, we sorted

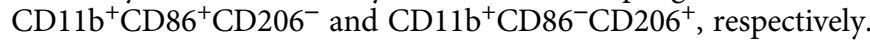
Our data showed that glioma-CM-pretreated ECs trend to induce alternative macrophage activation (Fig. 2b). To verify these results in human subjects, we pretreated human brain ECs with the CM harvested from the medium supernatant of cultured human GBM cells, and co-cultured these cells with human peripheral blood mononuclear cell (PBMC)-derived monocytes, followed by flow cytometry analysis. Consistent with the results from mouse studies, we showed that glioma-CM-pretreated human ECs stimulated human monocytes toward alternative activation, as evidenced by increased $\mathrm{CD} 206^{+} \mathrm{CD} 86^{+}$populations in $\mathrm{CD} 11 \mathrm{~b}^{+}$ cells (Fig. 2c). In addition, our data showed that glioma-CM alone slightly induced CD206 expression and macrophage alternative 
a

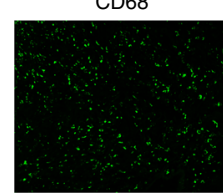

b

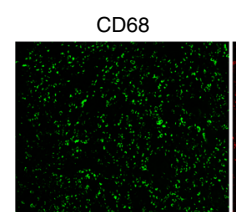

CD206

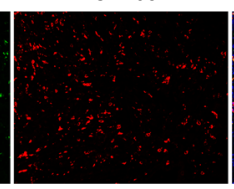

Immunofluorescence, CD68/CD206/Nuclei

CD163

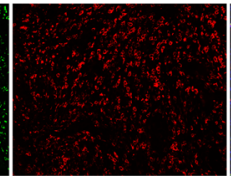

Immunofluorescence, CD68/CD163/Nuclei
Merge

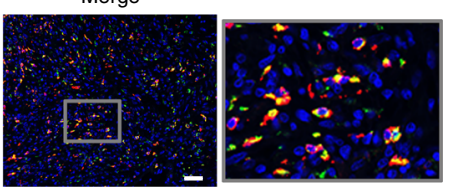

Merge

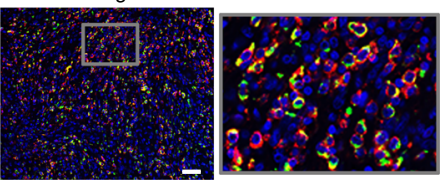

C

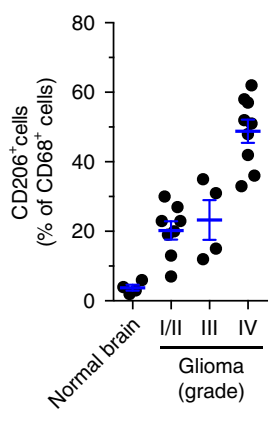

d
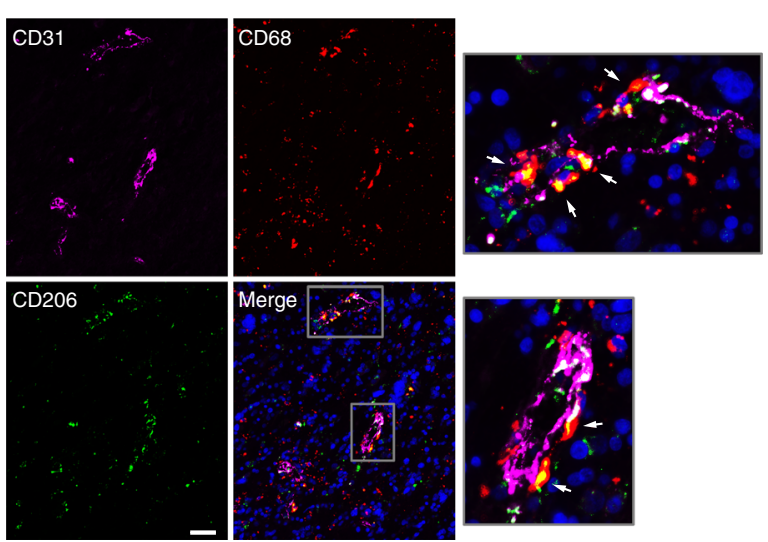

Immunofluorescence, CD31/CD68/CD206/Nuclei

e
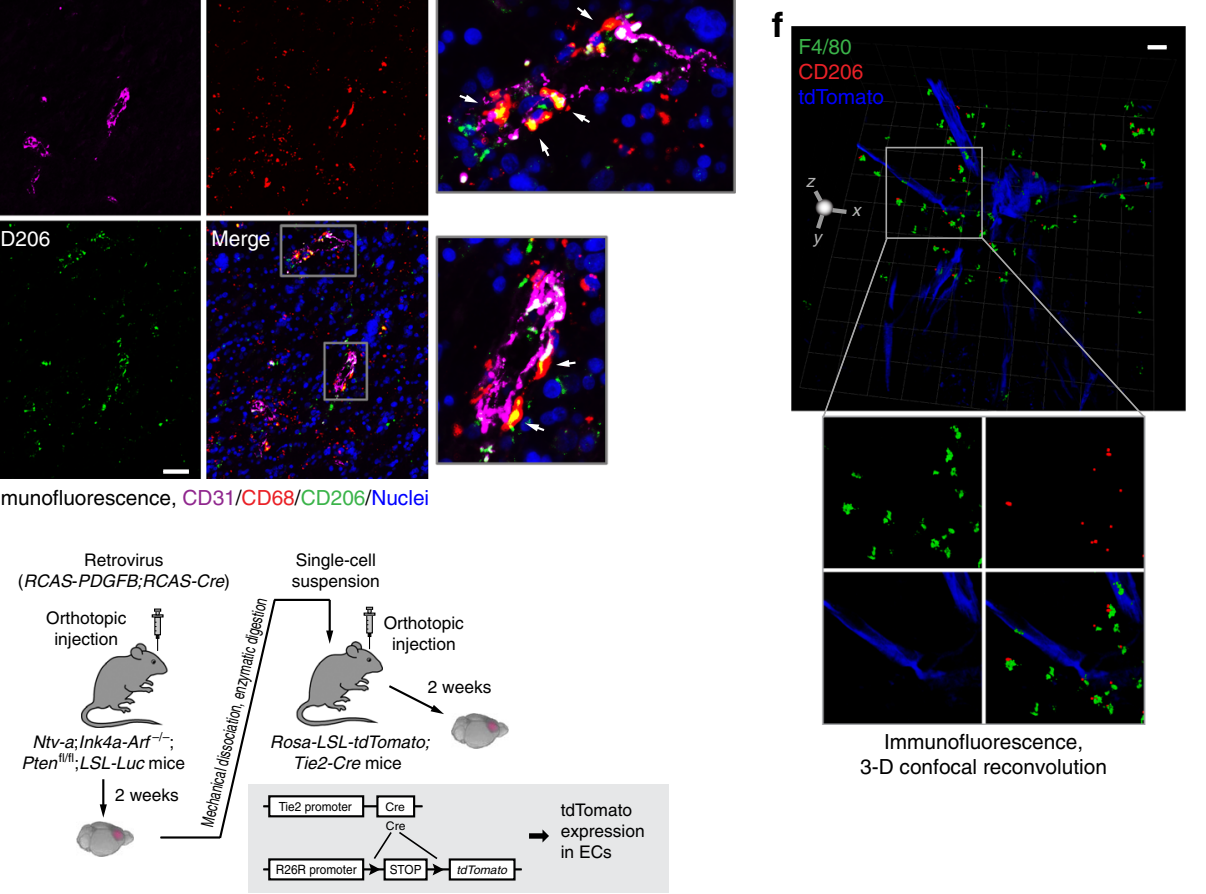

Fig. 1 Alternatively activated macrophages are localized proximately to GBM-associated ECs. a-d Tissue sections from human normal brain and surgical specimens of human glioma tumors were probed with different antibodies. a GBM tumor sections were stained with anti-CD68 and anti-CD206 antibodies. Representative images are shown ( $n=5$ GBM patient tumors). Bar represents $100 \mu \mathrm{m}$. Zoom-in factor: 4 . b GBM tumor sections were stained with anti-CD68 and anti-CD163 antibodies. Representative images are shown ( $n=5$ patient GBM tumors). Bar represents $100 \mu \mathrm{m}$. Zoom-in factor: 4 . c Normal brain and GBM tumor sections were stained with anti-CD68 and anti-CD206 antibodies. Quantified data are shown (total $n=4$ normal brains and 21 glioma tumors, mean \pm SEM). d GBM tumor sections were stained with anti-CD31, anti-CD206, and anti-CD68 antibodies. Representative images are shown ( $n=5$ patient GBM tumors). Arrows indicate CD68 ${ }^{+}$CD206 $6^{+}$cells. Bar represents $100 \mu \mathrm{m}$. Zoom-in factor: 4 . e, f GBM was induced by RCASmediated gene transfer in Ntv-a;Ink4a-Arf ${ }^{-/} ;$Pten $^{f / / f l} ;$ LSL-Luc mice, followed by orthotopic tumor transplantation into Rosa-LSL-tdTomato; Tie2-Cre mice. e Experimental procedure. f Thick sections were stained with anti-F4/80 and anti-CD206 antibodies, and subjected to confocal scanning imaging. 3-D images were generated and shown. Bar represents $200 \mu \mathrm{m}$. Zoom-in factor: 1.6

activation in human monocytes under normoxia, but moderately stimulated CD206 expression and alternative activation under hypoxia (Supplementary Fig. 4), implicating a possible role of hypoxia in macrophage polarization. However, hypoxia slightly promoted macrophage alternative activation but did not further enhance tumor EC-induced CD206 expression and alternative activation (Supplementary Fig. 5).

Moreover, PBMC-derived monocytes were co-cultured in transwells with normal brain ECs or GBM-associated ECs that were isolated from human GBM specimens. Immunoblot analysis shows that co-culture with GBM ECs induced robust arginase-1 expression in human monocytes (Fig. 2d), validating the stimulatory effects of tumor-associated ECs on alternative polarization of macrophages. Furthermore, PBMC-derived monocytes were directly co-cultured with brain ECs or GBMassociated ECs. Flow cytometry analysis showed that co-culture with GBM-derived ECs, but not normal ECs or CSF-1, induced robust $\mathrm{CD} 206$ expression in $\mathrm{CD}_{11 b^{+}}$macrophages (Fig. 2e). Finally, we analyzed anti-inflammatory cytokine IL-10 expression in these treated monocytes, as a function readout for alternative macrophage activation. Our data showed that co-culture with GBM ECs remarkably enhanced IL-10 expression in human monocytes (Fig. 2f), further verifying EC-mediated alternative macrophage activation in GBM. Taken together, these findings suggest a vascular niche for alternative polarization of macrophages in glioma microenvironment. 


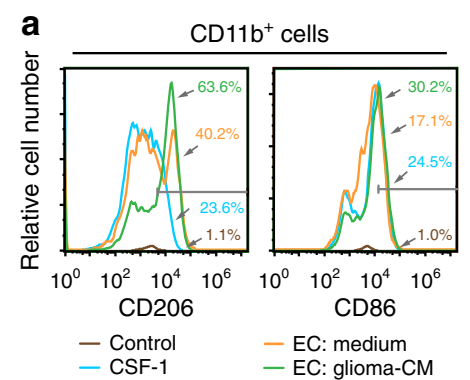

b
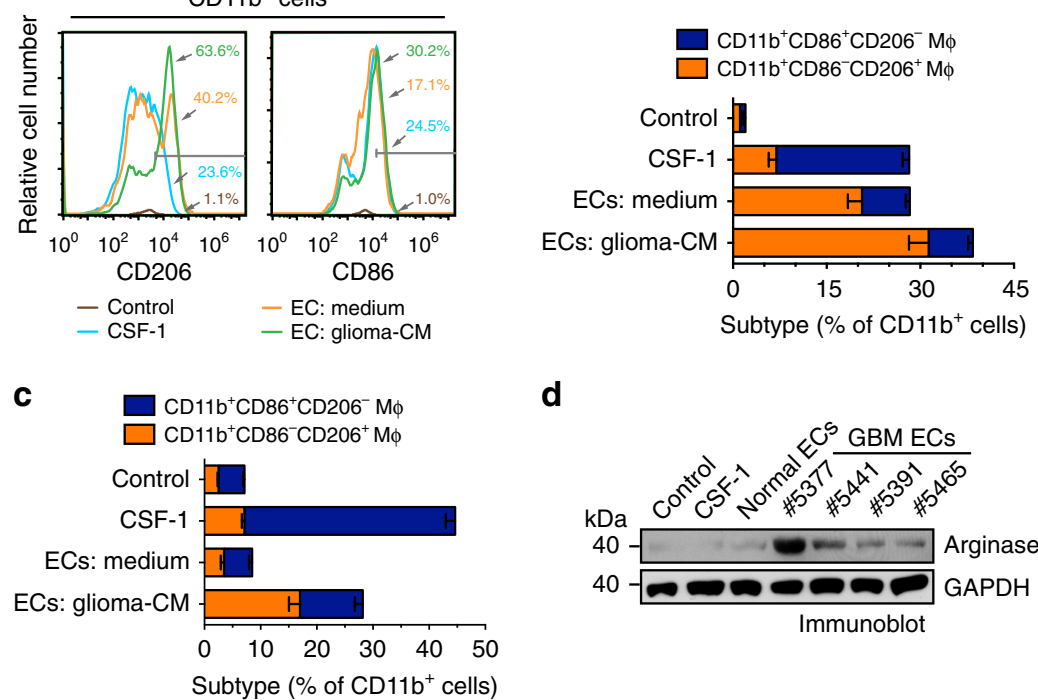

d
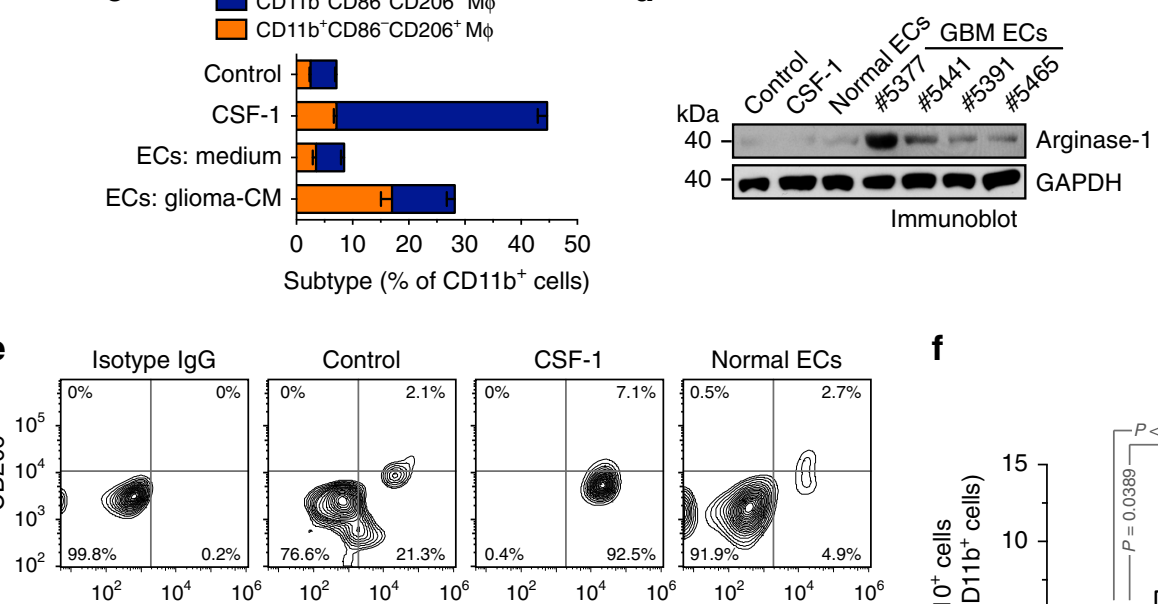

GBM ECs \#5377 GBM ECs \#5441 GBM ECs \#5391 GBM ECs \#5465

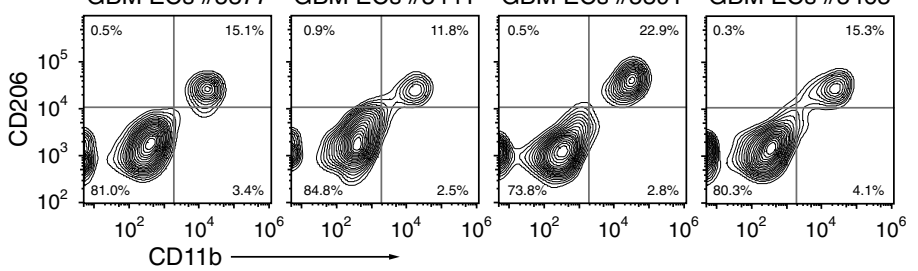

f

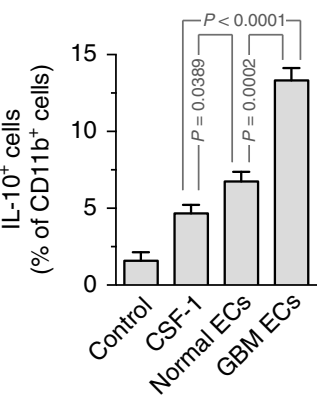

Fig. 2 GBM ECs induce alternative activation of macrophages. a, b Mouse brain microvascular ECs were pretreated with the glioma-conditioned medium (glioma-CM, harvested from medium supernatant of mouse GL26 glioma cells under 1\% hypoxia) or control medium for $24 \mathrm{~h}$. Mouse bone marrow (BM)derived macrophages were incubated with CSF-1 or co-cultured with pretreated ECs for 5 days, stained with anti-CD11b, anti-CD86, anti-CD206 antibodies, and analyzed by flow cytometry. a Representative results of CD206 and CD86 expression in CD11b ${ }^{+}$cells. b Quantified data in sorted CD11b ${ }^{+}$ macrophages $(M \phi, n=3-4$ mice, mean \pm SEM). c Human brain microvascular ECs were pretreated with the glioma-CM (harvested from medium supernatant of human U251 glioma cells under 1\% hypoxia) or control medium for $24 \mathrm{~h}$. Human peripheral blood mononuclear cell (PBMC)-derived monocytes were incubated with CSF-1 or co-cultured with pretreated ECs for 5 days, stained with anti-CD11b, anti-CD86, anti-CD206 antibodies, and subjected to flow cytometry analysis. Quantified data in sorted CD11b ${ }^{+}$cells $(n=3$, mean \pm SEM). d Human PBMC-derived monocytes were incubated for 5 days with CSF-1, or co-cultured with tumor-associated ECs isolated from different GBM patients or human normal brain microvascular ECs in upper and lower chambers of transwells, respectively. Monocytes were harvested and subjected to immunoblot analysis with anti-arginase-1 and anti-GAPDH antibodies. e Human PBMC-derived monocytes were incubated for 5 days with CSF-1, or co-cultured with tumor-associated ECs isolated from different GBM tumors ( $n=4$ patients) or human normal brain microvascular ECs. Cells were harvested, stained with anti-CD206, and anti-CD86 antibodies, and subjected to flow cytometry analysis. Representative images are shown. f PBMC-derived monocytes were incubated for 5 days with CSF-1, or co-cultured with tumor-associated ECs isolated from GBM patient \#5377 or human normal brain microvascular ECs. Cells were harvested, stained with anti-IL-10 and anti-CD11b antibodies, and subjected to flow cytometry analysis $(n=5$, mean \pm SEM). $P$ values were determined by Student's $t$ test

EC-secreted IL-6 induces macrophage alternative activation. We next investigated the mechanism(s) by which GBMassociated ECs induce macrophage alternative activation, initially focusing on EC secretion. Multiplex cytokine array analysis of medium supernatants indicated that glioma-CM remarkably altered EC expression of multiple cytokines and growth factors (Fig. 3a). Most robustly up-regulated cytokines included CCL5 and CXCL5, which regulate macrophage chemotaxis, and IL-6 and CSF-1, which have known functions acting on macrophages, particularly considering a recently published work showing a role of IL- 6 in macrophage alternative activation in diabetic inflammation ${ }^{26}$. Our data showed that
CCL5 and CXCL5 did not induce CD206 expression or macrophage alternative activation (Supplementary Fig. 6). Immunoblot analysis confirmed that glioma-CM remarkedly increased IL-6 expression and moderately increased CSF-1 expression in ECs (Fig. 3b), and also showed that GBMassociated ECs constitutively expressed IL-6 and CSF-1 at a higher level than normal brain ECs (Fig. 3c). Consistently with these in vitro results, our in vivo study with an orthotopic, syngenetic GL26 glioma model indicated a remarked increase in IL-6 expression by tumor-associated ECs, in comparison to normal brain ECs (Fig. 3d). Interestingly, IL-6, but not CSF-1, was preferentially localized in $\mathrm{CD} 31^{+}$ECs, implying that ECs as a major 
a
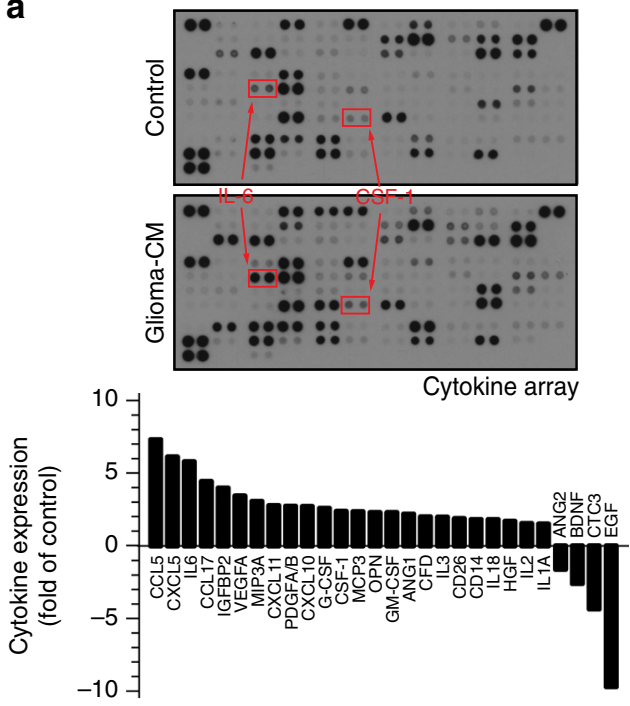

b

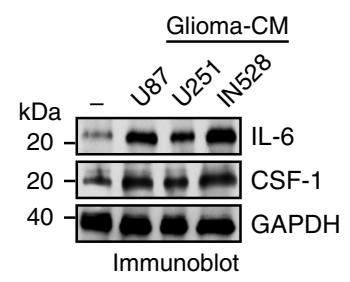

C

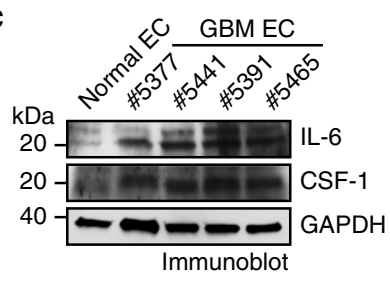

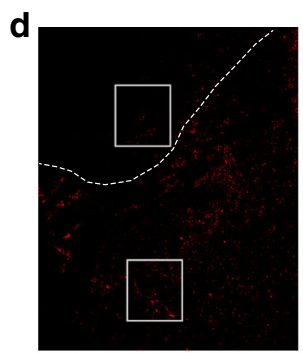
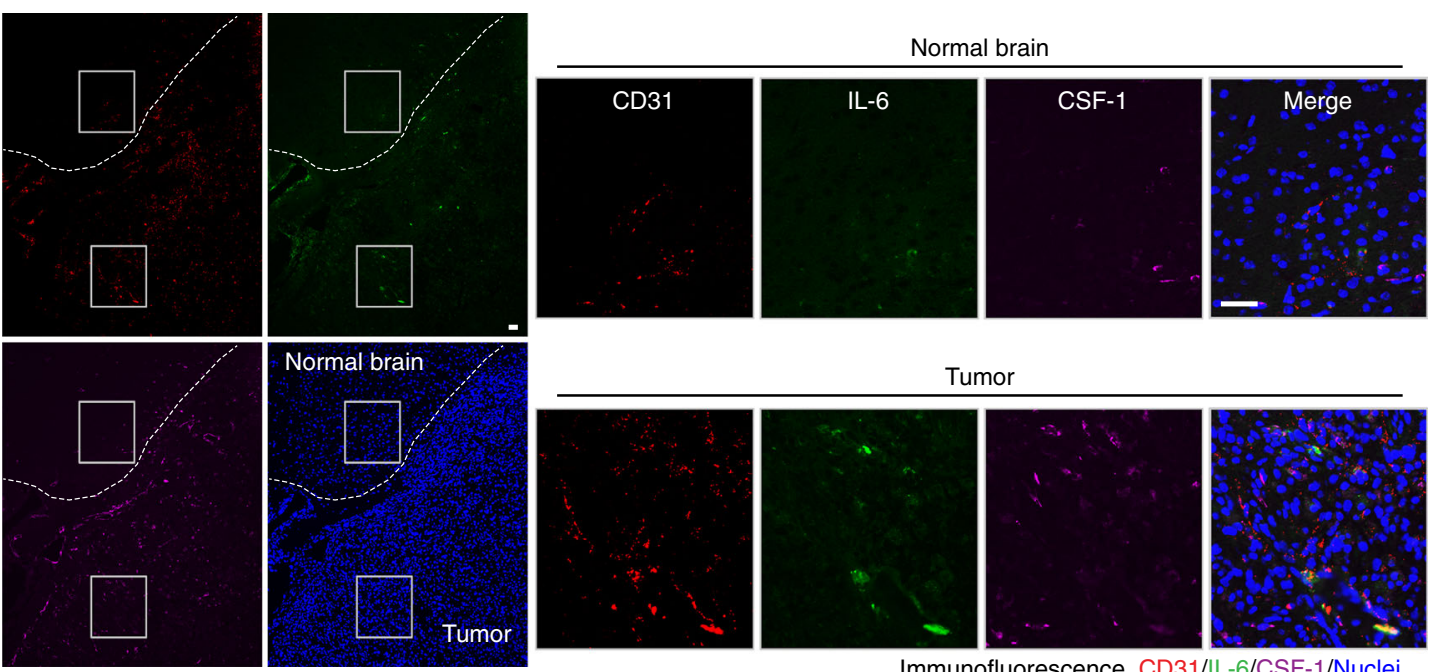

Immunofluorescence, CD31/IL-6/CSF-1/Nuclei

Fig. 3 GBM ECs express IL-6. a Human brain ECs were treated with glioma-CM for $24 \mathrm{~h}$, and cell lysates were subjected to multiplex cytokine array analysis. Left, a representative blot. Right, quantified dot intensity of most significantly changed cytokines. b Human microvascular brain ECs were treated with glioma-CM that were harvested from different human glioma cells. Cell lysates were immunoblotted. c Human microvascular brain ECs and tumorassociated ECs isolated from different GBM patients were subjected to immunoblot analysis. d Mouse GBM was induced by orthotopic injection of GL26 glioma cells into wild-type mouse. The brain sections that include normal brains and tumors were stained with anti-CD31, anti-IL-6, and anti-CSF-1 antibodies. Representative immunofluorescence images are shown. Right, enlarged area in normal and tumor tissues. Bar represents $50 \mu \mathrm{m}$. Zoom-in factor: 4

source for the expression of IL-6 but not CSF-1 in glioma microenvironment.

We next tested the role of IL- 6 in EC-induced macrophage alternative activation. Our data showed that both IL-6neutralizing and CSF-1-neutralizing antibodies reduced CD206 ${ }^{+}$ cell population in $\mathrm{CD}_{11 \mathrm{~b}^{+}}$mouse $\mathrm{BM}$ macrophages treated with glioma-CM-pretreated ECs, but did not affect $\mathrm{CD} 11 \mathrm{~b}^{+} \mathrm{CD} 86$ ${ }^{+} \mathrm{CD} 206^{-}$population (Fig. 4a, b), suggesting a crucial role of IL-6 and CSF-1 in EC-induced alternative macrophage activation. Consistent with this finding, small interfering RNA (siRNA)mediated knockdown of IL-6 in ECs inhibited EC-induced arginase-1 expression (Supplementary Fig. 7). Interestingly, treatment of mouse BM-derived macrophages (BMDMs) with purified IL- 6 moderately stimulated CD206 expression, but the combined treatment with IL- 6 and CSF-1 induced alternative polarization of macrophages, as evidenced by robustly enhanced $\mathrm{CD}_{206}{ }^{+}$population in $\mathrm{CD} 11 \mathrm{~b}^{+}$cells (Fig. 4c, d). Consistently, the combined treatment with IL-6 and CSF-1 increased CD206 population in human PBMC-derived monocytes (Supplementary Fig. 8). Furthermore, combined treatment with IL-6 and CSF-1, but not single treatment, remarkably induced arginase-1 expression (Fig. 4e). Together, these results suggest a role of EC-secreted IL-6, and possibly microenvironmental CSF-1, for macrophage alternative activation in GBM.

CSF-1 and IL-6 induce macrophage polarization through HIF$2 \alpha$. To explore the mechanisms by which CSF-1 and IL-6 induce arginase-1 expression and macrophage alternative activation, we performed a multiplex screening assay for the DNA-binding activity of 96 transcriptional factors in the treated macrophages. Unexpectedly, IL-6 treatment alone decreased the activities of most transcriptional factors (Fig. 5a). However, co-treatment of CSF-1 and IL-6 activated multiple transcriptional factors, 
a

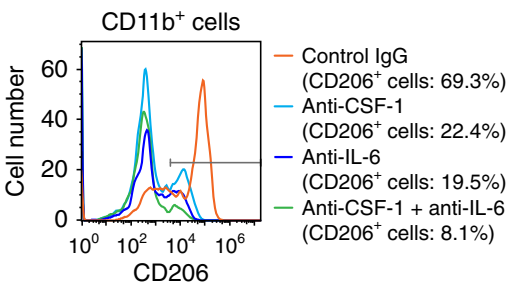

C

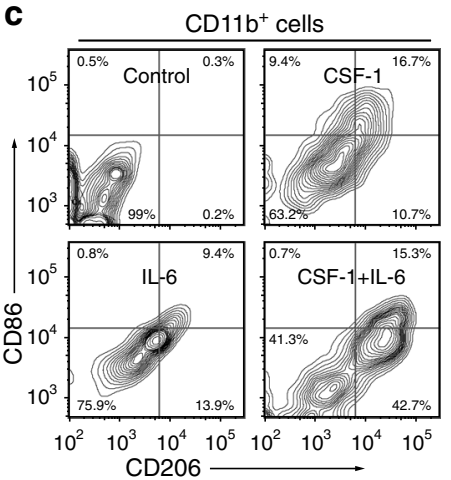

b

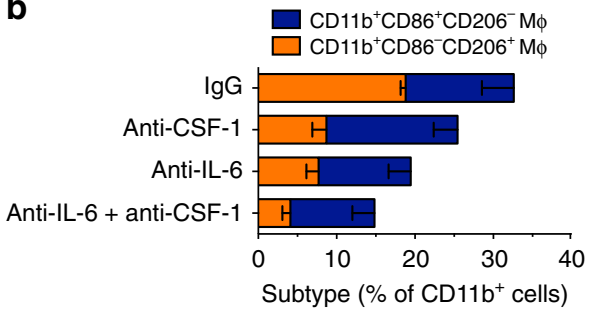

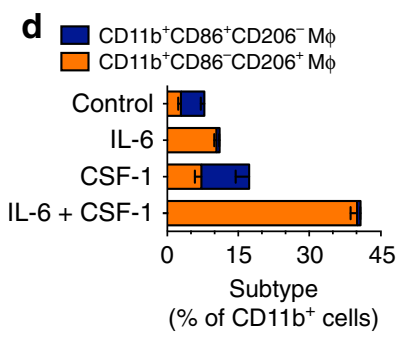

e

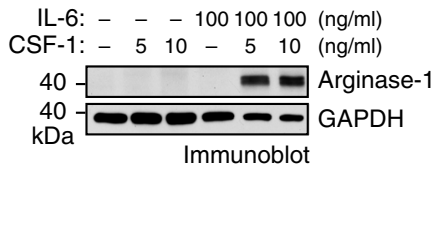

Fig. $4 \mathrm{IL}-6$ is critical for EC-induced macrophage alternative activation. a, b Mouse microvascular brain ECs were pretreated with the glioma-CM for $24 \mathrm{~h}$. Mouse BM-derived macrophages were co-cultured with pretreated ECs for 5 days in the presence of control IgG, anti-CSF- 1 antibody, or anti-IL- 6 antibody or both antibodies. The cells were stained with anti-CD11b, anti-CD86, anti-CD206 antibodies, and analyzed by flow cytometry. a Representative sorting for CD206 expression in CD11b ${ }^{+}$cells. b Quantified data in sorted CD11 b cells $(n=3-5$, mean \pm SEM). c-e Mouse BM-derived macrophages were treated with IL-6 and CSF-1 for 5 days. c, d The cells were stained with anti-CD11b, anti-CD86, and anti-CD206 antibodies, and analyzed by flow cytometry. c Representative sorting for CD206 and CD86 expression in CD11b ${ }^{+}$cells. $\mathbf{d}$ quantified data in sorted CD11b ${ }^{+}$cells $(n=3$, mean \pm SEM). e Cell lysates were immunoblotted

including AP1, HIF (hypoxia-inducible factor), KLF4, NF- $\mathrm{B}$, and PPAR (peroxisome proliferator-activated receptor) that have previously been shown critical for macrophage alternative activation $^{24,27}$. In contrast, CSF-1 treatment alone slightly activated HIF, KLF4, and PPAR, while inactivated AP1 and NF-кB. Notably, CSF-1 and IL-6 co-treatment remarkably increased the DNA-binding activity of HIF and PPAR, as indicated by about 70-fold and 80-fold increase in their activity, respectively.

We first tested the role of HIFs in macrophage alternative activation. HIFs are heterodimers comprising one of three major oxygen labile HIF- $\alpha$ subunits (HIF- $1 \alpha$, HIF- $2 \alpha$, or HIF- $3 \alpha$ ), and a constitutive HIF-1 $\beta$ subunit. HIFs are crucial mediators of hypoxic response, which transcribe a large number of genes that promote angiogenesis, anaerobic metabolism, and resistance to apoptosis, and therefore crucial for cancer development and progression $^{28-30}$. We verified the CSF-1- and IL-6-induced HIF transcriptional activation by HIF-responsive element (HRE)driven luciferase expression analysis (Fig. 5b). HIF- $1 \alpha$ and HIF$2 \alpha$ expression is known to be regulated by hypoxia-induced protein stability, but could also be induced by oxidative stress, growth factors, and succinate under normoxia ${ }^{31-35}$. Here we showed that CSF-1 and IL-6 remarkably increased protein expression of HIF- $2 \alpha$, but not HIF- $1 \alpha$, under normoxia in mouse macrophages (Fig. 5c) and human monocytes (Supplementary Fig. 9). Furthermore, the co-treatment stimulated expression and nuclei translocation of HIF-2 $\alpha$, but not HIF-1 $\alpha$ (Supplementary Fig. 10), supporting the increased HIF transcriptional activity and the distinct HIF- $2 \alpha$ expression induced by CSF- 1 and IL- 6 .

A recent study suggests a critical role of HIF- $2 \alpha$ in macrophage alternative activation ${ }^{36}$. Consistently, our data show that short hairpin RNA (shRNA)-mediated HIF-2 $\alpha$ knockdown almost completely blocked CSF-1-induced and IL-6-induced arginase-1 protein and mRNA expression (Fig. 5c, d) and macrophage alternative activation (Fig. 5e). In addition, shRNA-mediated knockdown of IL- 6 receptor- $\alpha$ and IL-6 neutralization significantly inhibited EC-CM-induced PPAR $\gamma, \mathrm{HIF}-2 \alpha$, and arginase- 1 expression, suggesting a critical role of IL-6 for activation of the HIF- $2 \alpha$ /arginase- 1 axis (Supplementary Fig. 11). Together, these data identify a critical role of HIF- $2 \alpha$ in CSF-1induced and IL-6-induced macrophage alternative activation.

PPAR $\gamma$ is required for HIF-2 $\alpha$ and arginase-1 expression. Interestingly, co-treatment with IL-6 and CSF-1, but not either one alone, remarkably enhanced protein and mRNA expression of HIF- $2 \alpha$ (Fig. $6 \mathrm{a}, \mathrm{b}$ ), suggesting that the co-treatment upregulates HIF- $2 \alpha$ expression through modulating HIF- $2 \alpha$ transcription. We analyzed the promoter sequence of HIF- $2 \alpha$ (EPAS1) and predicted that the transcriptional factors including SP1, PPAR $\gamma$, GATA-3, and HOXA9 may possibly bind to the region based on motif recognition pattern. We explored the regulatory mechanism for HIF- $2 \alpha$ transcription with a focus on PPAR $\gamma$, considering a robust activation of PPAR induced by the co-treatment (Fig. 5a) and an established role of PPAR $\gamma$ in macrophage alternative activation $^{37,38}$. Our data indicated that the co-treatment stimulated HIF- $2 \alpha$ promoter interaction with $\operatorname{PPAR} \gamma$, as shown by an immunoprecipitation analysis using synthetic biotin-labeled HIF$2 \alpha$ promoter DNA (Fig. $6 \mathrm{c}$ and Supplementary Fig. 12). Moreover, chromatin immunoprecipitation (ChIP) analysis validated that IL-6 and CSF-1 co-treatment induced HIF- $2 \alpha$ binding to PPAR $\gamma$ promoter in macrophages (Fig. 6d). Furthermore, shRNA-mediated knockdown of PPAR $\gamma$ inhibited IL- 6 and CSF1 co-treatment-induced expression of arginase-1 and HIF- $2 \alpha$ (Fig. 6e), suggesting that PPAR $\gamma$ regulates arginase- 1 and HIF- $2 \alpha$ expression in macrophages.

IL-6 induces inflammatory responses via JAK/STAT signaling and promotes cell survival and growth via MAPK-mediated and Akt-mediated pathway, respectively. CSF-1 is also known to be able to activate these survival and growth signaling pathways and also induces PPAR $\gamma$ activation. We investigated these signaling 

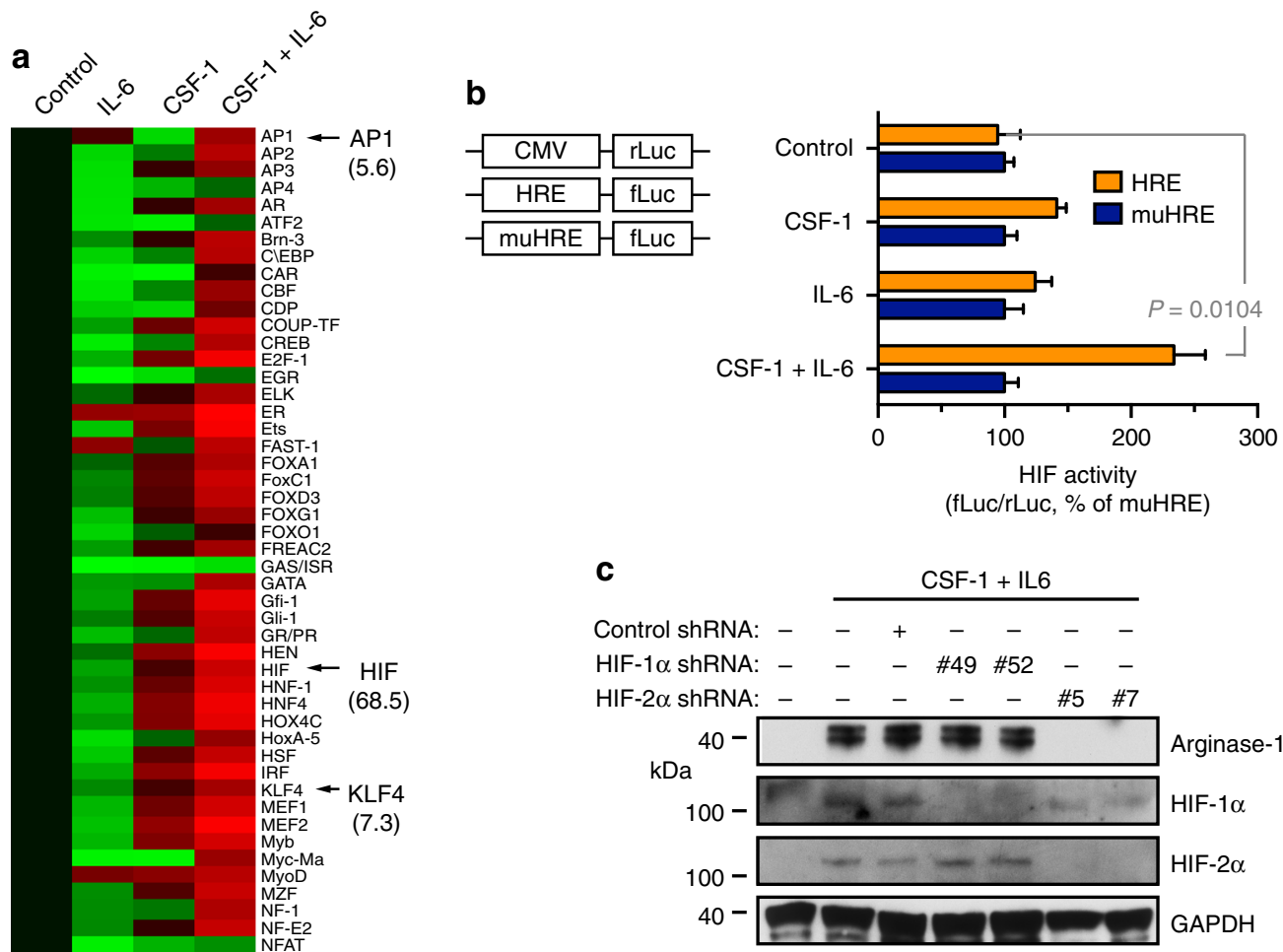

$\leftarrow$ KLF4

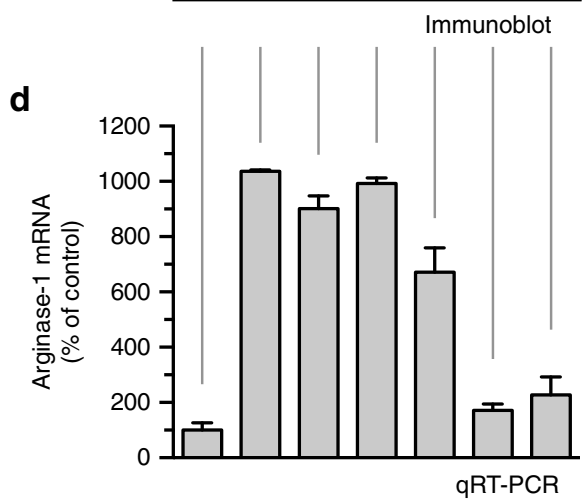

(81.0)

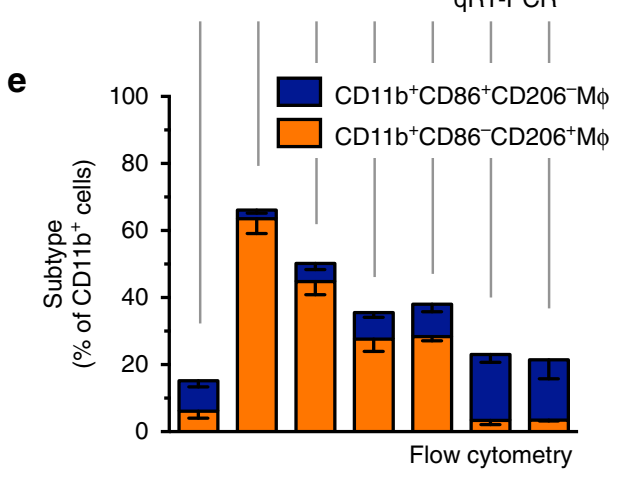

TF activity analysis
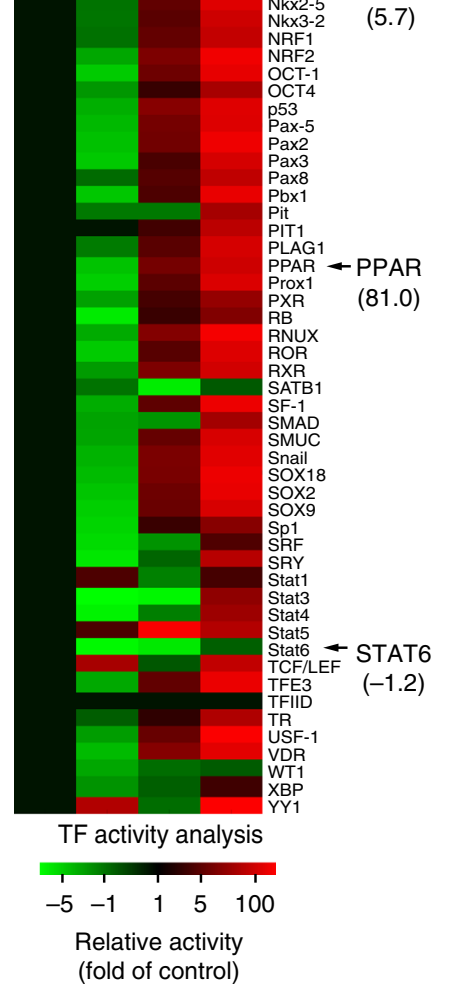

(81.0)

(fold of control)

Fig. 5 HIF-2 $\alpha$ is critical for IL-6-mediated arginase-1 expression and alternative macrophage activation. a Mouse BM-derived macrophages were treated with CSF-1 and IL-6 for 3 days. Nuclei proteins were subjected to multiplex profiling analysis for transcriptional factor activation. Activity was normalized with transcription factor IID, and expressed as the folds of control. b Mouse BM-derived macrophages were transduced with lentivirus that expresses CMV promoter-driven renilla luciferase (CMV-rLuc), hypoxia response element-driven firefly luciferase (HRE-fLuc), and mutated HRE-fLuc (muHRE-fLuc), followed by treatment with CSF-1 and IL- 6 for 2 days. Reporter activity radio of fLuc versus rLuc was determined by bioluminescence. Results were expressed as the percentage of muHRE $(n=3$, mean \pm SEM). P value was determined by Student's $t$ test. c-e Mouse BM-derived macrophages were transduced with lentivirus that expresses shRNAs targeting control scrambled sequence, HIF-1 $\alpha$ (\#49 and \#52) and HIF-2 $\alpha$ (\#5 and \#7), followed by treatment with CSF-1 and IL-6 for 10 days. c Cells were lysed and subjected to immunoblot analysis. d Arginase- 1 mRNA was analyzed by real-time RTPCR. Shown are quantified data (normalized with GAPDH expression, $n=3$, mean \pm SEM). e Cells were stained with anti-CD11b, anti-CD86, and antiCD206 antibodies, followed by flow cytometry analysis $(n=3$, mean \pm SEM) 
a

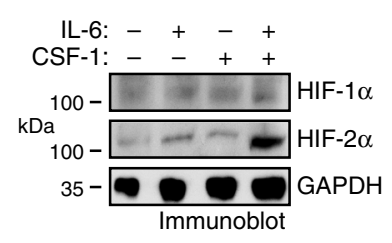

d
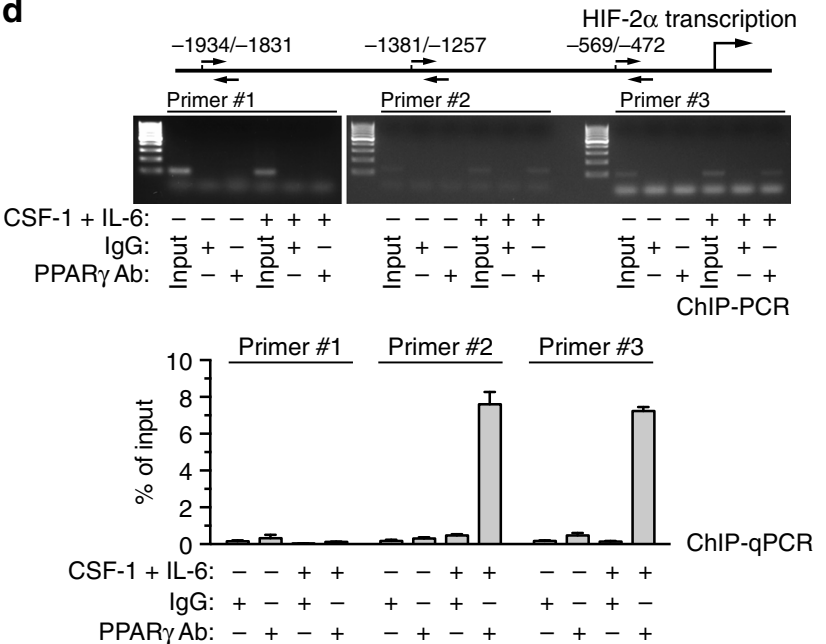

f

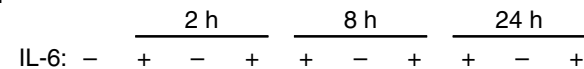

CSF-1: $-{ }_{-}+\ldots++\ldots+$

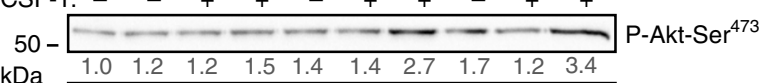

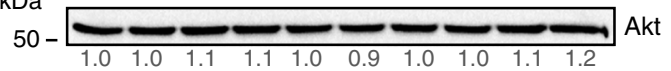

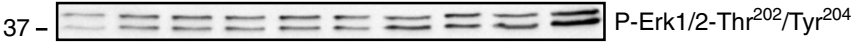
$\begin{array}{llllllllll}1.0 & 1.9 & 2.0 & 1.6 & 2.0 & 1.5 & 2.4 & 2.2 & 2.5 & 5.1\end{array}$

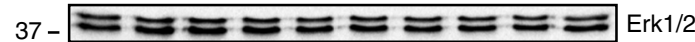

\begin{tabular}{llllllllll}
\hline 1.0 & 1.0 & 1.0 & 0.9 & 0.8 & 0.8 & 0.8 & 0.8 & 0.7 & 0.9
\end{tabular}

250 - $\begin{array}{lllllllll} & & \end{array}$ \begin{tabular}{llllllllll}
1.0 & 1.5 & 1.5 & 1.6 & 1.6 & 1.5 & 3.4 & 1.0 & 0.7 & 3.7 \\
\hline
\end{tabular}

$250-\longrightarrow-\infty=0-m$ mTOR $\begin{array}{llllllllll}1.0 & 0.9 & 1.0 & 1.0 & 1.0 & 1.8 & 1.0 & 1.0 & 1.0 & 1.0\end{array}$

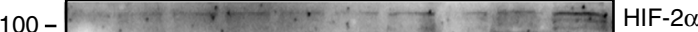

\begin{tabular}{|llllllllll|}
\hline 1.0 & 1.3 & 1.8 & 2.1 & 1.6 & 0.8 & 1.8 & 1.1 & 2.4 & 4.2 \\
\hline
\end{tabular}

$37-\square=\square=\square$ Arginase-1

$\begin{array}{llllllllll}1.0 & 0.7 & 0.9 & 0.9 & 1.0 & 0.9 & 1.6 & 1.0 & 1.0 & 3.9\end{array}$

$37-\longrightarrow$ GAPDH

$\begin{array}{llllllllll}1.0 & 1.0 & 1.0 & 1.0 & 1.0 & 1.0 & 1.0 & 1.0 & 1.0 & 1.0\end{array}$

Immunoblot, relative fold of band density

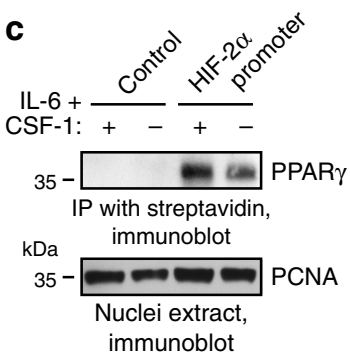

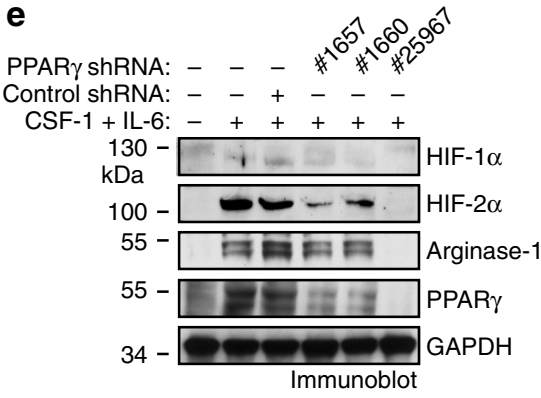
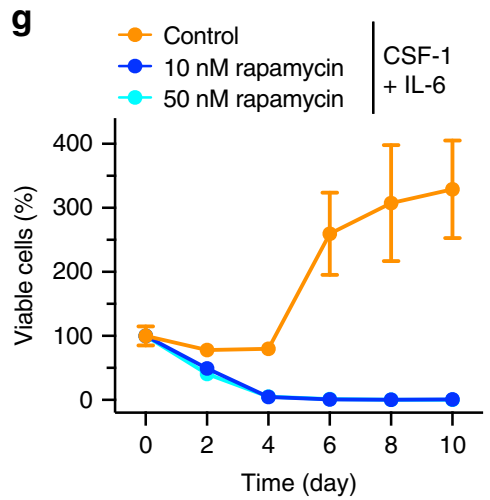

Fig. 6 PPAR $\gamma$ induces HIF-2 $\alpha$ and arginase- 1 expression in macrophages. a, b Mouse BM-derived macrophages were treated with or without CSF-1 and IL-6 for 5 days. a Cell lysates were immunoblotted. b mRNA was extracted and subjected to quantitative RT-PCR analysis. Results were normalized with GAPDH level and expressed as folds of control $(n=3$, mean \pm SEM). $P$ value was determined by Student's $t$ test. $\mathbf{c}$, d Mouse BM-derived macrophages were treated with or without CSF-1 and IL-6 for 3 days. c Nuclei protein was incubated with biotin-labeled synthetic DNAs that encode control scrambled or HIF-2 $\alpha$ promoter sequence, followed by immunoprecipitation with streptavidin-conjugated beads. Precipitants and nuclei protein were immunoblotted. d Nuclei extracts were subjected to chromatin immunoprecipitation (ChIP) analysis. Immunoprecipitants with control IgG or anti-PPAR $\gamma$ antibody were analyzed by PCR and electrophoresis (upper) or by quantitative PCR (bottom, $n=3$, mean \pm SD). e Mouse BM-derived macrophages were transduced with lentivirus that expresses shRNAs targeting control scrambled sequence and PPAR (\#1657, \#1660, and \#25967), followed by treatment with CSF-1 and IL6 for 10 days. Cells were lysed and subjected to immunoblot analysis. f Mouse BM-derived macrophages were treated with or without CSF-1 and IL-6. At different time points post-treatment, cells were lyzed and subjected to immunoblot analysis. Band density was quantified. $\mathbf{g}$ Mouse BM cells were pretreated with or without rapamycin, followed by incubation with CSF-1 and IL-6. Cell viability was determined ( $n=3$ mice, mean \pm SEM) 
events with time resolution in macrophages co-treated with IL-6 and CSF-1. Co-treatment with CSF-1 increased IL-6-induced Akt1, Erk, and mTOR signaling activation (Fig. 6f). Furthermore, treatment with LY294002 and rapamycin, pharmacological inhibitors of PI3K/Akt and mTOR, respectively, or shRNAmediated PPAR $\gamma$ knockdown, inhibited IL-6-induced and CSF-1induced macrophage proliferation (Fig. $5 \mathrm{~g}$ and Supplementary Fig. 13), suggesting that Akt/mTOR/PPAR $\gamma$ is required for IL-6induced and CSF-1-induced macrophage growth. Together, we propose a model for co-treatment-induced signaling mechanism for macrophage alternative activation: co-treatment induces arginase- 1 expression through PPAR $\gamma$ and HIF- $2 \alpha$, leading to macrophage alternative activation, and simultaneously it activates Aktl/mTOR to promote survival and growth of the alternatively activated macrophages in the tumor microenvironment.

ECs are one of major sources of IL-6 expression in GBM. Previous immunohistochemial studies with human GBM specimens suggest tumor-associated ECs and inflammatory cells as the major sources for IL-6 expression in the tumor microenvironment $^{39}$. Consistently, we showed that GBM-associated ECs expressed IL-6 at a relatively higher level, compared with U251 glioma cells, T4123 glioma stem cells, and human PBMC monocytes (Supplementary Fig. 14). To rigorously investigate the in vivo role of ECs in IL-6 expression as well as IL-6's functions in GBM progression, we generated a conditional IL-6 knockout Cdh5-Cre ${ }^{\mathrm{ERT} 2} ; \mathrm{Il} 6^{\mathrm{fl} / \mathrm{fl}}$ mouse line, in which IL-6 knockout was induced by tamoxifen-inducible Cre ${ }^{\text {ERT2 }}$ expression under ECspecific Cdh5 promoter (Fig. 7a). The efficient and selective knockout was validated by immunoblot and quantitative reverse transcription-PCR (RT-PCR) in aortic and brain ECs (Fig. 7b, c and Supplementary Fig. 15). We took advantage of the orthotopic, genetic murine GBM model with a native microenvironment, induced by $R C A S / N$-tva-mediated somatic PDGF gene transfer in Ink4a-Arf ${ }^{-1-} ;$ Pten $^{-/-}$neural stem/progenitor cells (Fig. 7d), followed by tumor transplantation into the new generated $C d h 5-C r e^{\mathrm{ERT} 2} ; I l 6^{\mathrm{f} / \mathrm{fl}}$ mice. Notably, the genetic GBM mouse model is immunocompetent, which recapitulates the major features of human GBM including pseudopalisading necrosis and microvascular proliferation ${ }^{35}, 40$. Immunoblot analysis of these tumors reveals that IL-6 deletion in ECs significantly reduced IL-6 expression in whole tumor lysates (Fig. 7e), confirming that ECs are one of major sources of IL- 6 expression in GBM microenvironment.

Endothelial IL-6 is critical for GBM growth and progression. We finally investigated the in vivo role of endothelial IL-6 in GBM progression. Our data show that IL-6 deletion in ECs significantly improved animal survival in the mice bearing the genetically induced GBM tumors, leading to an increase in overall survival time by $45 \%$ (19 versus 27.5 days, in control and tamoxifen-treated Chd5-Cre ${ }^{\mathrm{ERT} 2} ;$ Il $6^{\mathrm{f} / \mathrm{fl}}$ mice, Fig. 8a). Notably, about $20 \%$ of mice survived through the experimental process, showing no detectable tumors when euthanized at day 50. Consistent with the critical role of endothelial IL-6 in mouse survival, EC-specific knockout of IL-6 significantly reduced tumor growth, as indicated by a $70 \%$ decrease in average tumor volume (at day 12 after tumor implantation, Fig. 8b). Furthermore, IL-6 knockout eliminated pseudopalisades (P) and microvascular proliferation (MP) in the tumors (Fig. 7c), suggesting a critical role of ECderived IL-6 in GBM progression.

Strikingly, IL-6 knockout robustly induced extensive necrosis and leukocyte infiltration (Fig. 8c), possibly due to its negative effects on macrophage-mediated tumor immunity. Consistently, our data showed more neutrophils and $\mathrm{CD}^{+} \mathrm{T}$ cells infiltrated in the tumors (Supplementary Fig. 16). Flow cytometry analysis of tumor-associated $\mathrm{F} 4 / 80^{+}$macrophage cells show that indicated that IL-6 knockout robustly decreased $\mathrm{CD}^{-} 6^{-} \mathrm{CD} 206^{+}$ a
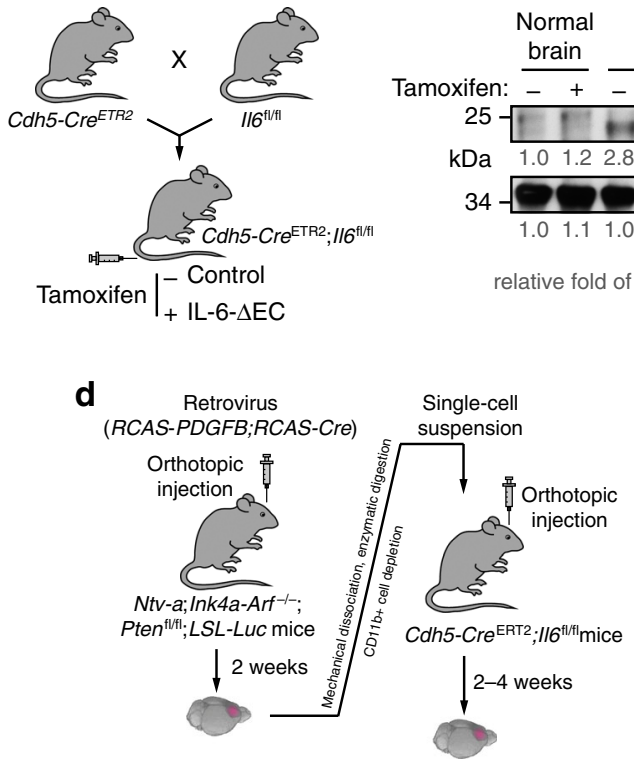

b

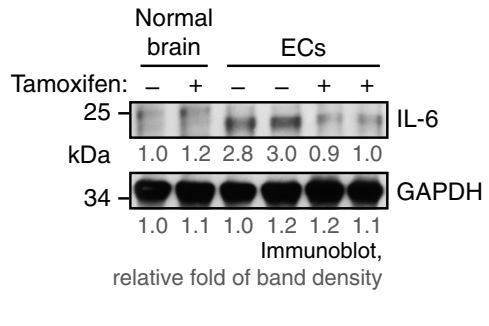

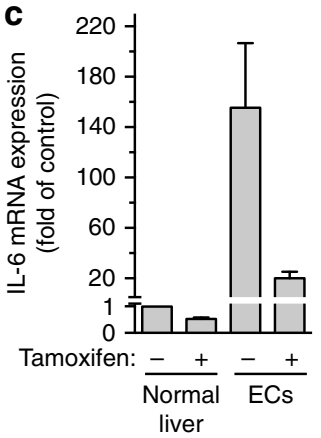

e

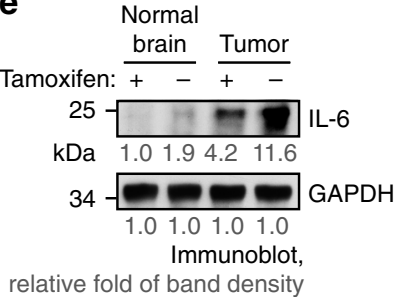

Fig. 7 ECs are a major source for IL-6 expression in GBM. a-c Cdh5-Cre $\mathrm{ERT2}^{\mathrm{ER}} / 16^{\mathrm{fl} / \mathrm{fl}}$ mice were generated by crossing Cdh5-Cre ${ }^{\mathrm{ERT} 2}$ mice with $/ 16^{\mathrm{fl} / \mathrm{fl}}$ mice. Mice ( 2 weeks old) were injected with tamoxifen for consecutive 5 days to induce EC-specific IL- 6 knockout. a Schematic approach. b, c ECs were isolated from mouse aortas. $\mathbf{b}$ Brain tissue and ECs were subjected to immunoblot analysis. Band density was quantified. $\mathbf{c}$ mRNA was extracted and subjected to quantitative RT-PCR analysis. Results were normalized with GAPDH level and expressed as folds of control $(n=3$, mean \pm SEM). d, e The primary GBM in

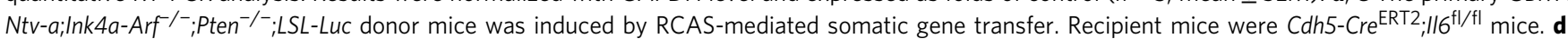
Schematic approach. e Normal brain and tumor tissues were homogenized. Tissue lysates were immunoblotted. Band density was quantified 
a

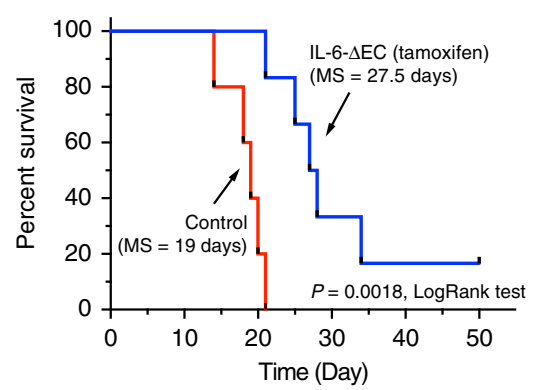

d

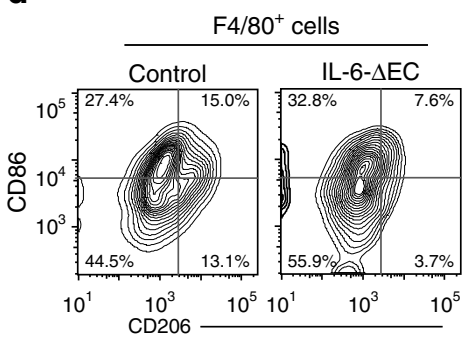

b

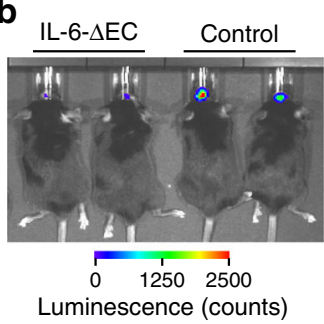

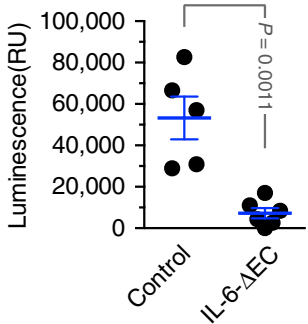

C

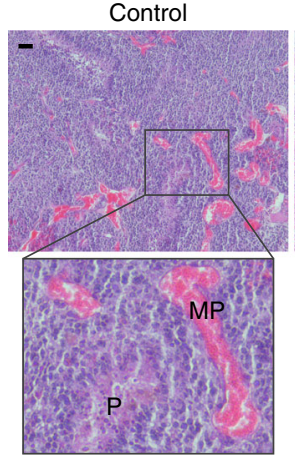

g
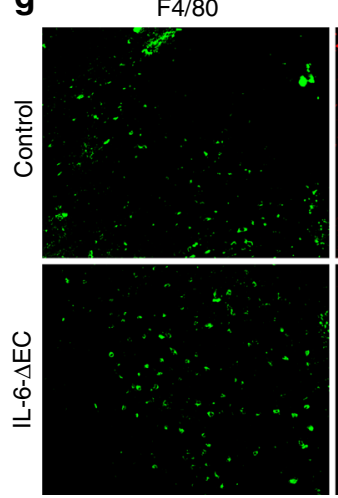

IL-6- $\Delta \mathrm{EC}$

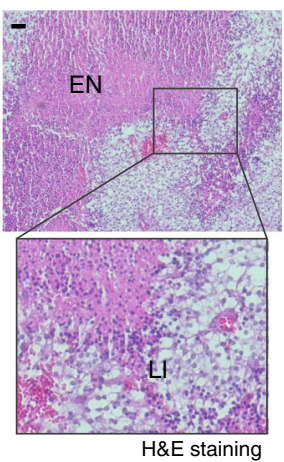

iNOS
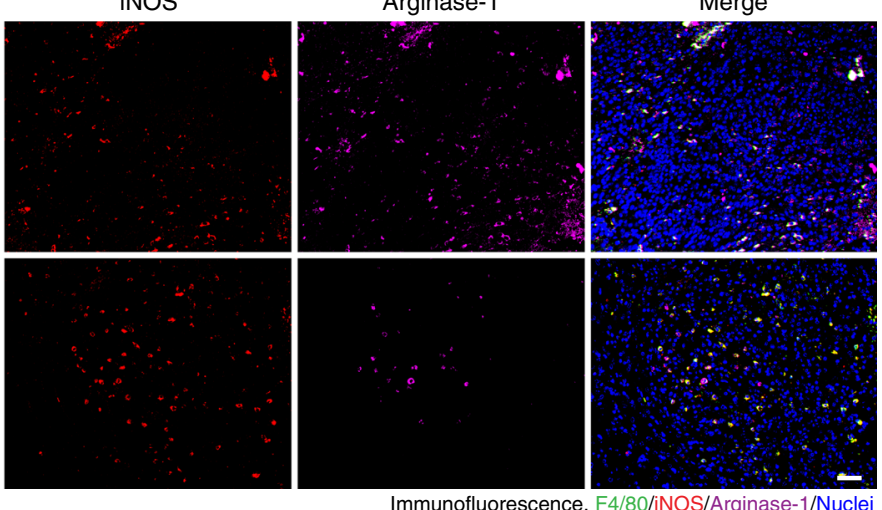

Immunofluorescence, F4/80/iNOS/Arginase-1/Nuclei e

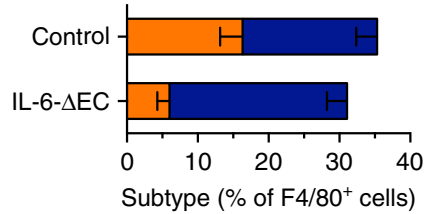

$\mathbf{f}$

$\mathrm{F} 4 / 80^{+} \mathrm{IL}-12^{+} \mathrm{IL}-10^{-} \mathrm{M} \phi$

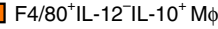

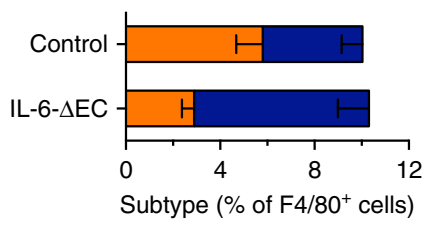

Fig. 8 Endothelial IL-6 is critical for macrophage alternative activation and GBM growth and progression. The genetically engineered GBM model was

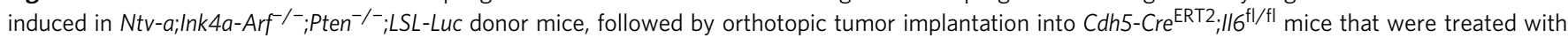
(IL-6- $\Delta \mathrm{EC}$ ) or without (Control) tamoxifen. a Animal survival was monitored for 50 days post-injection ( $n=5-6$ mice, one representative result from three independent experiments). $P$ values were determined by log-rank (Mantel-Cox) tests. MS, median survival. b Tumor growth was analyzed by bioluminescence. Left, representative images. Right, quantitative analysis of integrated luminescence in tumors at day 12 (mean \pm SEM, $n=5-6$, one representative result from three independent experiments). $P$ value was determined by Student's $t$ test. c Tumor sections were stained with hematoxylin and eosin (H\&E). Representative images are shown $(n=10$ mice). P pseudopalisades, MP microvascular proliferation, EN extensive necrosis, LI leukocyte infiltration. Bar represents $100 \mu \mathrm{m}$. Zoom-in factor: 3. e, f Tumors were excised. Single-cell suspensions were prepared and subjected to flow cytometry analysis. d, e Single-cell suspensions were probed with anti-F4/80, anti-CD86, and anti-CD206 antibodies. CD206 and CD86 expression were analyzed in sorted F4/80+ cells. $\mathbf{d}$ Representative sorting. e Quantified results (mean $\pm \mathrm{SEM}, n=10-14$ mice). $\mathbf{f}$ Single-cell suspensions were probed with anti-F4/80, anti-IL-10, and anti-IL-12 antibodies. IL-10 and IL-12 expression was analyzed in sorted F4/80 cells. Show are quantified results (mean \pm SEM, $n=8-13$ mice). $\mathbf{g}$ Tumor sections were stained and analyzed by immunofluorescence. Tumor sections were probed with anti-iNOS, anti-arginase-1, anti-F4/80 antibodies ( $n=10$ mice). Bar represents $100 \mu \mathrm{m}$ 


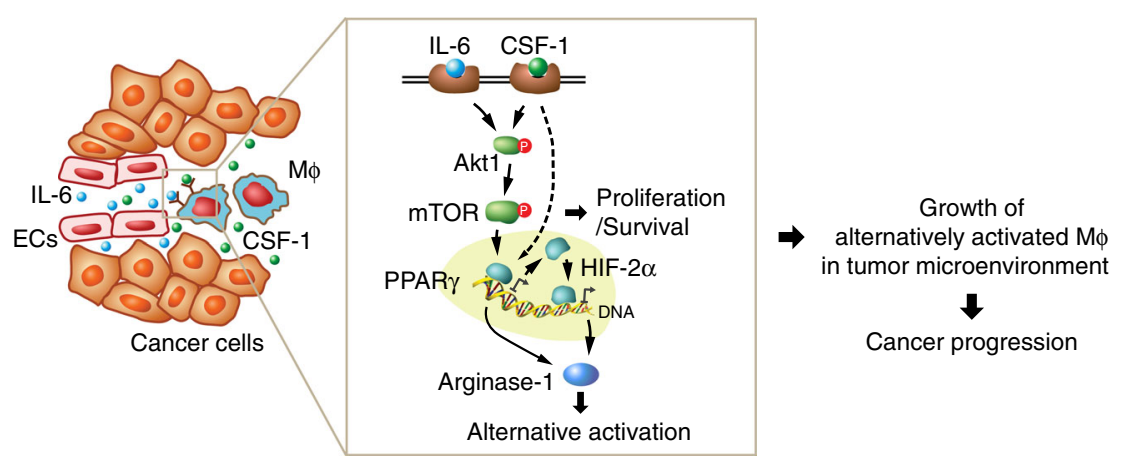

Fig. 9 A schematic model. In glioma microenvironment, endothelial cell-derived IL-6 and microenvironmental CSF-1 synergistically activate downstream Akt1/mTOR pathway and induces transcriptional activation of PPAR $\gamma$ in macrophages (M $\phi$ ), in turn leading to HIF-2 $\alpha$-mediated arginase-1 expression, and inducing macrophage alternative polarization. The activation of mTOR also induces cell proliferation, contributing to cell survival and growth of alternatively activated macrophages, eventually leading to glioma progression

macrophage population and slightly increased $\mathrm{CD}^{+} 6^{+} \mathrm{CD} 206^{-}$ macrophage population (Fig. 8d, e). Moreover, IL-6 deletion in ECs increased the expression of proinflammatory IL-12 and reduced the expression of anti-inflammatory IL-10 (Fig. 8f). Futhermore, IL-6 deletion did not affect iNOS expression, but remarkedly inhibited arginase-1 expression in tumor-associated F4 $/ 80^{+}$macrophages (Fig. $8 \mathrm{~g}$ ), supporting the important role of endothelial IL- 6 in macrophage alternative activation. Together, these data suggest that endothelial cell-derived IL- 6 is critical for macrophage alternative polarization and GBM progression.

\section{Discussion}

Our studies identify a vascular niche that drives macrophage alternative polarization and GBM progression. Namely, tumorassociated ECs express and release IL-6, jointing with CSF-1 in the microenvironment, induces HIF- $2 \alpha$-dependent arginase- 1 expression through activation of PPAR- $\gamma$, leading to macrophage alternative polarization and GBM progression. IL-6 and CSF-1 also induce mTOR activation, resulting in cell survival and growth of alternatively activated macrophages in the tumor microenvironment (Fig. 9).

Macrophages are a crucial player in tumor-host immune interaction and cancer development and progression. Functional polarization, for example, M0 (naïve status), M1 (classically activated), or M2 (alternatively activated), of macrophages represents a key mechanism that controls their functions, switching roles between tumor suppressing and promoting $4,5,41$. Although it has been well established that most of gliomaassociated macrophages acquire alternative activation to execute tumor-promoting and tumor-immunosuppressive functions ${ }^{23,42}$, recent ex vivo studies based on transcriptome analysis of cytokine and cell marker expression in isolated myeloid cells suggest that glioma-associated macrophages exhibit a M0 polarization profile ${ }^{43}$, suggesting that classical or alternative activation of macrophages depends on the stimulus from tumor microenvironment and also are possibly not driven by cell plasticity-mediated permanent cell fate transition.

Vascular ECs are a major component of tumor microenvironment. In addition to their classical functions for delivering oxygen and nutrients to support tumor growth, tumor-associated ECs act as a niche that fuels tumor growth, progression, and metastasis by producing paracrine factors, that is, angiocrines, to tumor microenvironment ${ }^{40,44-52}$. For example, it is well known that perivascular niche is critical for stemness maintenance and self-renewal in cancer stem cells ${ }^{44,47,50}$. The role of vascular niche in tumor immunity regulation, nevertheless, remains unidentified. Here our study reveals that EC-derived IL- 6 promotes macrophage alternative activation and GBM progression in vivo, therefore identifying a vascular niche that controls macrophage functions in cancer. Supportive to this concept, a recent study shows that in vitro co-culture with ECs induces differentiation of hematopoietic progenitor and stimulates macrophage alternative activation $^{53}$.

Previous work shows that genetic IL- 6 ablation blocks tumor formation of spontaneous mouse glioma driven by glial fibrillary acidic protein-mediated expression of viral Src oncogene ${ }^{54}$. Our work reveals that EC-derived IL- 6 is critical for tumor growth and progression in a genetically induced GBM mouse model. Importantly, analyses of TCGA and Rembrandt databases show that elevated IL-6 expression correlates with poor overall survival in glioma and GBM patients (Supplementary Fig. 17). Consistently, IL-6 blockade treatment significantly improved animal survival time by $35 \%$ in our genetically engineered GBM model (17 versus 23 days, in control and anti-IL-6 antibody-treated mice, Supplementary Fig. 18). Thus, IL-6 blockade may serve as an efficient strategy for therapeutic intervention of GBM. In addition, IL-6-deficient mice develop normally ${ }^{55}$. As such, its ECpreferential induction in tumor microenvironment, but dispensability in development, suggests that IL-6 may present a highly selective and non-noxious therapeutic target for cancer treatment.

Alternative macrophage activation is known to be driven by multiple cytokines including IL- 4 and IL-13 6 , 22 . Here we identify IL-6 as a major driver for alternative macrophage activation in glioma microenvironment. IL- 6 has classically been recognized as a proinflammatory cytokine 56,57 . As such, IL-6 receptor blockade has been served as an important therapeutic strategy for the treatment of inflammatory diseases including rheumatoid arthritis ${ }^{58}$. Interestingly, IL-6 regulates $\mathrm{T}$ cell proliferation and survival $^{56,59}$, induces IL- 4 production by $\mathrm{CD} 4^{+} \mathrm{T}$ cells ${ }^{60}$, and promotes $\mathrm{T}$ cell Th17 differentiation ${ }^{61}$. A recent study reports that IL-6 induces IL-4 receptor expression in macrophages, leading to their alternative activation in diabetic inflammation ${ }^{26}$, suggesting an unexpected role for IL- 6 in macrophage-mediated anti-inflammatory responses. Likewise, our data for the first time identify IL-6 as a critical instigator of macrophage alternative activation in cancer. We show that IL- 6 and CSF- 1 induce robust arginase-1 expression that primes macrophages to alternative activation. In addition, previous studies reveal that IL- 6 enhances expression of IL-4 and IL-13, two stimulators for macrophage alternative activation, likely secreted by $\mathrm{T}$ cells ${ }^{60}$, which may further promote macrophage alternative activation in the tumor microenvironment.

Macrophage polarization is subjected to transcriptional regulation driven by cues in the tissue microenvironment ${ }^{27}$. Activation of multiple transcriptional factors, mainly induced by IL-4 
and IL-13, drives macrophage alternative activation, which includes AP1, HIF- $2 \alpha$, KLF4, PPAR $\gamma$, and STAT- $6^{27,} 37,38,62,-64$. The mechanisms that control macrophage activation in cancer, particularly induced by IL-6, remain largely unknown. Here we reveal that IL-6 and CSF-1 induce PPAR $\gamma$-dependent HIF- $2 \alpha$ transcription, leading to arginase- 1 expression and macrophage alternative activation in GBM. Interestingly, a recent work that has screened 270 transcriptional factors shows that PPAR $\gamma$ coactivator/estrogen-related receptor induces HIF- $2 \alpha$ expression in neuroblastoma, independent of canonical hypoxia-mediated regulation of protein stability, but through transcription regulation $^{65}$, supporting our observation of HIF- $2 \alpha$ mRNA expression induced by PPAR $\gamma$ in GBM.

In summary, our study identifies a vascular niche for the regulation of tumor immunity and reveals an IL-6-mediated mechanism controlling macrophage alternative activation and GBM progression. Specifically, IL-6 and CSF-1 induce PPAR $\gamma$ dependent HIF-2 $\alpha$ transcription, leading to arginase-1 expression and macrophage alternative polarization in GBM. Thus, targeting IL-6 may offer exciting therapeutic opportunities to reactivate macrophage-mediated tumor immunity, which may block tumor progression and treatment resistance.

\section{Methods}

Patient and tumor endothelial cell sorting. All patients received surgery at the Department of Neurosurgery of the University of Pennsylvania and were enrolled in a single institution tissue banking protocol that was approved by the University of Pennsylvania Human Studies Committee. Consent was obtained from all patients. Tumor-associated ECs were isolated as previously described ${ }^{66}$. In brief, tumor-derived single-cell suspensions were prepared by the tissue bank. Red cells were removed with ACK lysis buffer (Life Technologies). Cell suspension was subjected to magnetic activating cell sorting (MACS) with anti-CD31 antibodyconjugated magnetic beads (Miltenyi Biotech, 130-091-935). Sorted ECs were verified by Dil-Ac-LDL (Bioquote) absorption, and over $99 \%$ of cells were Dil-AcLDL-positive.

Cell culture. Human and mouse brain microvascular ECs (ScienCell and PromoCell) were maintained in Endothelial Cell Medium (ECM, ScienCell) at $37^{\circ} \mathrm{C}$ in a humidified air atmosphere with $5 \% \mathrm{CO}_{2}$. All cells were used between passages 2 and 5. GL26 mouse glioma cells were kindly provided by Chunsheng Li (University of Pennsylvania) and cultured in Dulbecco's modified Eagle's medium (DMEM) medium (Gibco) supplemented with 5\% fetal bovine serum (FBS). Chicken DF-1 fibroblasts (ATCC) were maintained in DMEM medium (ATCC, 30-2002) containing $5 \% \mathrm{FBS}$ at $39^{\circ} \mathrm{C}$ in a humidified air atmosphere with $5 \% \mathrm{CO}_{2}$. T4123 glioma stem cells were kindly provided by Jeremy Rich (Cleveland Clinic) and then cultured in serum-free Neurobasal-A medium (Gibco), supplemented with B-27 Supplement Minus Vitamin A (Gibco), GlutaMax (Gibco), sodium pyruvate (Gibco), fibroblastic growth factor ( $5 \mathrm{ng} / \mathrm{ml}$, R\&D Systems), and EGF $(20 \mathrm{ng} / \mathrm{ml}$, $\mathrm{R} \& \mathrm{D}$ Systems). All cancer cell lines were checked and showed no mycoplasma contamination.

Mice. Wild-type (WT) mice on the C57BL/6J background were obtained from

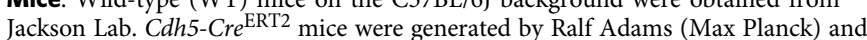
kindly provided by Nancy Speck (University of Pennsylvania) and Bisen Ding $(\text { Cornell })^{67}$. Il6 ${ }^{\mathrm{fl} / \mathrm{fl}}$ mice have been described previously ${ }^{68,69}$. Cdh5-Cre ${ }^{\mathrm{ERT} 2} ; \mathrm{Il} 6^{\mathrm{fl} / \mathrm{fl}}$ mice were generated by crossing $I l 6^{\mathrm{fl} / \mathrm{fl}}$ mice with $\mathrm{Cdh} 5-\mathrm{Cr} \mathrm{e}^{\mathrm{ERT} 2}$ mice. Mice were genotyped with primers including IL-6 FP: 5'-CCCACCAAGAACGATAGTCA-3', and IL-6 RP: 5'-GGTATCCTCTGTGAAGTCCTC-3'. Cdh5-Cre ${ }^{\mathrm{ERT}} ;{ }^{\prime}$ Il6 ${ }^{\mathrm{fl} / \mathrm{fl}}$ and $I l 6^{\mathrm{fl} / \mathrm{fl}}$ mice ( 2 weeks old) were intraperitoneally injected with $0.1 \mathrm{ml}$ of $5 \mathrm{mg} / \mathrm{ml}$ tamoxifen daily for consecutive 5 days. Rosa-LSL-tdTomato;Tie2-Cre mice were generated by crossing Rosa-LSL-tdTomato mice (Jackson Lab) with Tie2-Cre mice (Jackson Lab). All animal studies were reviewed and approved by the Institutional Animal Care and Use Committees (IACUC) at the University of Pennsylvania. All animals were housed in the Association for the Assessment and Accreditation of Laboratory Animal Care-accredited animal facility of the University of Pennsylvania.

Isolation and culture of mouse ECs. Mouse aortic ECs were isolated from Cdh5$C r e^{\mathrm{ERT} 2} ; I l 6^{\mathrm{fl} / \mathrm{fl}}$ mice as previously described ${ }^{35}$. In brief, thoracic aorta was isolated from 3-week-old mice and cut into pieces. Aortic rings were embedded in phosphate-buffered saline (PBS)-prewashed Matrigel and then incubated in DMEM/F-12 medium containing 5\% FBS for 5 days. After rinsing with PBS, the rings were removed and the remaining cells were incubated with $2 \mathrm{U} / \mathrm{ml}$ Dispase I (Gibco, 17105-041) for $20 \mathrm{~min}$ at $37^{\circ} \mathrm{C}$. After centrifugation at $500 \times \mathrm{g}$ for $10 \mathrm{~min}$, the cell pellets were washed with PBS. ECs were also isolated from mouse brain. Single-cell suspension was prepared and subjected to MACS with anti-CD31 antibody-conjugated magnetic beads (Miltenyi Biotech, 130-091-935). Cells were cultured in DMEM/F-12 medium supplemented with $25 \mu \mathrm{g} / \mathrm{ml}$ EC growth supplement (Sigma) and 5\% FBS. All cells were used between passages 2 and 4 .

Genetic GBM mouse model. GBM was induced in mice as previously described ${ }^{66}$ In brief, chicken DF-1 fibroblasts (ATCC) were transfected with RCAS-PDGF-B and RCAS-Cre plasmids to produce retrovirus, and orthotopically injected into Ntv-a;Ink4a-Arf ${ }^{-1-} ; P_{t e n}{ }^{\mathrm{f} / \mathrm{fl}} ; L S L-l u c$ mice to induce GBM through RCAS/n-tvamediated gene transfer. Tumors were isolated and subjected to mechanical dissociation with a gentleMACS Dissociator (Miltenyi Biotech) and enzymatic digestion with collagenase II and dispase II to obtain single-cell suspensions. About 8 weeks old $C d h 5-C r e^{\mathrm{ETR} 2} ; I l 6^{\mathrm{fl} / \mathrm{fl}}$ and $I l 6^{\mathrm{fl} / \mathrm{fl}}$ mice, half male and half female, were orthotopically and stereotactically injected with $10^{5}$ GBM tumor cells that express luciferase. Tumor growth was monitored by whole-body bioluminescence using an IVIS 200 Spectrum Imaging System after retro-orbital injection of luciferin (150 $\mathrm{mg} / \mathrm{kg}$, GoldBio). Survival was monitored for 50 days. For antibody treatment experiment, tumor-bearing mice were intraperitoneally treated with anti-CSF-1 (BioXcell, BE0204, $1 \mathrm{mg}$ per mouse x 4) or anti-IL-6 (BioXcell, BE0046, $200 \mu \mathrm{g}$ per mouse $\mathrm{x} 4$ ) antibody or control rat immunoglobulin G (IgG) (BioXcell, BE0090, $200 \mu \mathrm{g}$ per mouse $\mathrm{x} 4$ ). Mice were euthanized when exhibiting severe GBM symptoms including domehead, hemiparesis, or more than $20 \%$ of body weight loss. Mice were randomized to receive treatment, and the investigators were not blinded.

GL26 glioma mouse model. GL26 glioma was induced in mice as previously described $^{66}$. In brief, about 8 weeks old Rosa-LSL-tdTomato;Tie2-Cre and WT mice were orthotopically and stereotactically injected with $10^{5}$ GL26 mouse glioma cells that express luciferase. Tumor growth was monitored by whole-body bioluminescence using an IVIS 200 Spectrum Imaging System after retro-orbital injection of luciferin $(150 \mathrm{mg} / \mathrm{kg}$, GoldBio).

Preparation of glioma-CM. Mouse GL26 glioma cells or Human U251, U87, and IN528 glioma cells were cultured with DMEM medium supplemented with 5\% FBS. Cells at about $90 \%$ confluence were exposed to hypoxia $\left(1 \% \mathrm{O}_{2}\right)$ in a humidified air atmosphere at $37^{\circ} \mathrm{C}$ for $24 \mathrm{~h}$. Culture medium were centrifuged at $5,000 \times g$ for $30 \mathrm{~min}$ to remove cellular debris and then the supernatant was collected.

Preparation of EC-CM. Human normal brain microvascular ECs (ScienCell and PromoCell) and GBM patients' tumor-derived ECs (\#5377, \#5441, \#5391, \#5465) were maintained with ECM (ScienCell). When reaching about $70 \%$ confluence, cells were cultured at $37^{\circ} \mathrm{C}$ for $24 \mathrm{~h}$. Culture medium were centrifuged at 5,000 $\times \mathrm{g}$ for $30 \mathrm{~min}$ to remove cellular debris and then the supernatant was collected.

BMDM isolation and treatment. Freshly isolated femur and tibia from WT C57BL/6 mice were flushed with RPMI-1640 medium (Life Technologies). Cells were harvested and passed through a $40-\mu \mathrm{m}$ strainer. Red cells were removed with ACK lysis buffer. BM cells were cultured in RPMI-1640 medium supplemented with $5 \%$ FBS (Life Technologies). Cells were incubated with $10 \mathrm{ng} / \mathrm{ml}$ human CSF1 (BioLegend, 574808) for 7 days to induce macrophage differentiation. Cells were treated with $100 \mathrm{ng} / \mathrm{ml}$ mouse IL-6 (BioLegend, 575708) and CSF-1 for different times.

Human peripheral blood-derived monocytes and treatment. Primary human monocytes, purchased from the Human Immunology Core at the University of Pennsylvania, were isolated from healthy volunteer donors following leukapheresis by negative selection. All specimens were collected under a University Institutional Review Board-approved protocol, and written informed consent was obtained from each donor. Isolated cells were incubated in RPMI-1640 medium supplemented with $5 \%$ heat-inactivated FBS. Cells were treated with $10 \mathrm{ng} / \mathrm{ml}$ human CSF-1, 100 $\mathrm{ng} / \mathrm{ml}$ human IL-6 (BioLegend, 570808), and normal human ECs or patient tumor EC-CM.

Co-culture of BM cells and ECs and treatment. Mouse BM cells were co-cultured with mouse brain microvascular ECs, CCL5 (PeproTech, 250-07), or CXCL5 (PeproTech, 300-22) under normoxia or hypoxia $\left(1 \% \mathrm{O}_{2}\right)$. ECs were seeded in 6well plates $\left(5 \times 10^{3} /\right.$ well $)$ and then cultured overnight with DMEM medium containing $5 \%$ FBS, followed by treatment with glioma-CM as described above. ECs were washed with PBS and co-cultured with freshly prepared BMDMs $\left(10^{4}\right.$ cells per well) for 5 days in RPMI-1640 media supplemented with 5\% FBS. Cells were treated with specific neutralizing antibodies against CSF-1 $(1 \mu \mathrm{g} / \mathrm{ml}, \mathrm{R} \& \mathrm{D}$ Systems, AF416) and IL-6 (1 $\mu \mathrm{g} / \mathrm{ml}$, BioLegend, 504505), or control IgG (Sigma). Cells were fixed and subjected to immunofluorescence analysis. Single-cell suspension was prepared by using Versene solution $(0.02 \%$ EDTA, Thermo) and then subjected to flow cytometry analysis. 
shRNA and siRNA treatment. The $293 \mathrm{~T}$ cells (ATCC) were co-transfected with packaging vectors (System Biosciences) and lentiviral expression vectors that encode shRNA targeting HIF- $1 \alpha$ and HIF- $2 \alpha$ (kindly provided by Celeste Simon as previously published ${ }^{70}$ ), and PPAR $\gamma$ (Sigma, TRCN0000001657,

TRCN0000001660, and TRCN0000025967) for $8 \mathrm{~h}$. For IL-6R- $\alpha$ knockdown, the oligonucleotides (\#1: sense, $5^{\prime}$-TACCGACCTGTATGGTCAAATTCAAGAGAT TTGACCATACAGGTCGGTTTTTTTC-3' , and antisense, $5^{\prime}$-TCGAGAAAAAAA CCGACCTGTATGGTCAAATCTCTTGAATTTGACCATACAGGTCGGTA-3'; \#2: sense: $5^{\prime}$-TATCAGTACGAAAGTTCTACTTCAAGAGAGTAGAACTTTCGT ACTGATTTTTTTC-3', antisense, 5'-TCGAGAAAAAAATCAGTACGAAAGTTC TACTCTCTTGAAGTAGAACTTTCGTACTGATA-3'; \#3: sense, 5'-TCAATAC CGTAAACCACAGCTTCAAGAGAGCTGTGGTTTACGGTATTGTTTTTTC-3', antisense, 5'-TCGAGAAAAAACAATACCGTAAACCACAGCTCTCTTGAAG CTGTGGTTTACGGTATTGA-3') that encode shRNAs were synthesized and cloned into the pSicoR lentivirus expression vector, followed by transfection with 293T cells. After change with fresh medium and incubation for $48 \mathrm{~h}$, medium supernatants containing lentivirus were collected. Freshly separated BMDMs were infected with lentivirus in the presence of $8 \mu \mathrm{g} / \mathrm{ml}$ polybrene (Millipore). Stably shRNA-expressing cells were selected with $1.8 \mu \mathrm{g} / \mathrm{ml}$ puromycin and maintained in the culture medium with $0.5 \mu \mathrm{g} / \mathrm{ml}$ puromycin. Cells were transfected with control or IL-6 siRNA (Ambion, 4390771) by using Lipofectamine 2000 (Invitrogen).

Dual luciferase reporter assay. WT or mutated HRE with firefly luciferase reporter in pGL2 (kindly provided by Celeste Simon) were subcloned into lentivirus expression vector pCDH (System Biosciences). The 293T cells (ATCC) were co-transfected with packaging vectors (System Biosciences) and lentiviral expression vectors that express HRE-luciferase or control CMV-renilla luciferase. Freshly prepared BMDMs were treated and transduced with lentivirus, followed by measurement of luciferase activity using a Dual Luciferase Assay System (Promega). Each measured firefly luciferase activity was normalized by the renilla luciferase activity in the same well.

Transcription factor activation profiling array. BMDMs were treated with different cytokines. Nuclear proteins were isolated with a nuclear extraction kit (Signosis, SK-0001), and analyzed by using a 96-well plate transcription factor (TF) activation array (Signosis, FA1002) according to the manufacturer's instruction. The activity of each transcriptional factor was normalized as the fold of TFIID's activity.

\section{Chromatin immunoprecipitation. ChIP assays were performed using a Magna} ChIP kit (Millipore, MAGNA0001) according to the manufacturer's instructions. Mouse BM cells $\left(10^{7}\right.$ cells) cultured in 15-cm dishes were treated with CSF-1 and IL-6. Cells were crosslinked with $1 \%$ formaldehyde for $10 \mathrm{~min}$ at room temperature, followed by glycine incubation for $5 \mathrm{~min}$. The cells were sonicated three times, each for $16 \mathrm{~s}$, using a W-385 sonicater (Heat Systems Ultrasonics). Immunoprecipitants with chromatin were prepared by using $20 \mu \mathrm{g}$ anti-PPAR $\gamma$ (Santa Cruz, sc-7196) or anti-normal rabbit IgG (Santa Cruz, sc-2027) with cell lysates from $10^{6}$ cells in $0.5 \mathrm{ml}$ reaction buffer. The primers pairs for HIF-2 $\alpha$ promoter used in the ChIP-PCR and ChIP-qRT-PCR are listed as followings: Primer \#1: FP, 5'-TACAG CTCAAATCCAGCAGAAGC-3', RP, 5'-GAGGGAGGGAAAGACCAGACAATAA-3'; Primer \#2: FP, 5'-GCTTTCTCCGCAATTCACAACTATG-3', RP, 5'-TCT TAGACACTTTCCCATTTCCTACTT-3'; Primer \#3: FP, 5'-CGCCATTACTCAG TCCTGCGCTAA-3', RP, 5'-TTCCGCAGAACTGCGACTTGTTT-3'.

Cytokine array. Human brain microvascular ECs were treated with glioma-CM or control medium for $24 \mathrm{~h}$ at $37^{\circ} \mathrm{C}$. After washing twice with PBS, cells were incubated with DMEM/F-12 medium supplemented with $2 \%$ bovine serum albumin (Sigma) for $12 \mathrm{~h}$. Cell lysates were subjected to multiplex cytokine analysis using a Human XL Cytokine Array (Ary022, R\&D Systems) according to the manufacturer's instructions.

Quantitative RT-PCR analysis. Total RNA was isolated using TRIzol reagent (Invitrogen) from treated mouse BMDMs, mouse aortic ECs, and mouse liver tissue. RNA was reversely transcribed using SuperScript III First-Strand Synthesis SuperMix (Life Technologies), and subjected to real-time PCR analysis using TaqMan ${ }^{\circledR}$ Fast Universal PCR Master Mix (Life Technologies) and primers: HIF-1 $\alpha$ (Applied (Biosystems, Mm00468869_m1), HIF-2 $\alpha$ (EPAS1, Applied Biosystems, Mm00438717_m1), IL-6 (Applied Biosystems, Mm00446190_m1), and arginase-1 (FP: 5'-CTCCAAGCCAAAGTCCTTAGAG-3'; RP: 5'-GCATCCACCCAAATGA CACAT-3'). Quantitative RT-PCR was performed in a 20- $\mu$ l reaction volume using Fast SYBR Green Master Mix (Applied Biosystems) with 18S rRNA or glyceraldehyde 3-phosphate dehydrogenase (GAPDH) used as endogenous controls.

Immunofluorescence and histology. Human surgical specimens from subjects with glioma or normal brain tissues (from US Biomax, BioChain, and from patients treated in Department of Neurosurgery, Sun Yat-sen University Cancer Center, China. Patient specimens were collected under a University Institutional Review Board-approved protocol, and written informed consent was obtained from each patient), or mouse tumors tissues were used. Paraffin sections were de-paraffinized and rehydrated, and subjected to antigen retrieval in Target Retrieve Solution (Dako, S1699) at $95^{\circ} \mathrm{C}$ for $20 \mathrm{~min}$. Sections were blocked with $5 \%$ horse serum for $1 \mathrm{~h}$ at room temperature. Human samples were incubated with anti-CD31 (1:100, Cell Signaling, 3528), anti-CD68 (1:100, Cell Signaling, 76437), anti-CD86 (1:100 BD Pharmingen, 555656), anti-CD163 (1:100, Serotec/Bio-Rad, MCA1853T), antiCD206 (1:100, Santa Cruz, SC376108), anti-arginase-1 (1:100, Santa Cruz, SC20150), or anti-iNOS (Abcam, ab3523) antibody overnight at $4{ }^{\circ} \mathrm{C}$. For mouse tissues, sections were incubated with anti-CD31 (1:100, Dianova, DIA310), anti-IL6 (1:100, Dako, A0082; Novus, NB600-1131), anti-CSF-1 (1:100, Millipore, AB5320), anti-F4/80 (1:100, Miltenyi Biotech, 130-102-379), anti-arginase-1 (1:100, Santa Cruz, SC-18354), anti-Mac3 (1:100, BD Pharmingen, 550292), anti-CD3 (1:100, Abcam, ab11089), anti-CD8 (1:100, Cell Signaling, 98941), or antineutrophil (1:100, Cedariane, CL8993B) antibody overnight at $4^{\circ} \mathrm{C}$. For cell culture, cells were fixed with $4 \%$ paraformaldehyde for $10 \mathrm{~min}$ and incubated with anti-CD11b (1:100, BioLegend, 101206), anti-arginase-1 (1:100, Santa Cruz, sc18354), or anti-iNOS (Abcam, ab3523) antibody. Sections were stained with Alexa Fluor $^{\circledast}$ 488-conjugated, 568-conjugated, and 647-conjugated appropriate secondary IgGs (1:500, Life Technologies) for $1 \mathrm{~h}$ at room temperature. Images were acquired with an AxioImager fluorescence microscope (Zeiss) equipped with AxioCam 506 CCD camera (Zeiss). For histological study, sections were stained with hematoxylin and eosin (H\&E), and imaged with an AxioLab microscope (Zeiss) equipped with AxioCam HRC CCD camera (Zeiss).

Brain tissue preparation and 3-D confocal imaging. The Rosa-LSL-tdTomato; Tie2-Cre mice bearing RCAS-induced tumors were perfused with $30 \mathrm{ml}$ PBS and $10 \mathrm{ml} 4 \%$ paraformaldehyde. The brains were dissected and fixed in $4 \%$ paraformaldehyde for $24 \mathrm{~h}$ at $4{ }^{\circ} \mathrm{C}$. Hydrogels were prepared by using $40 \%$ acrylamide and $10 \%$ VA-044 in PBS. The brain was soaked in the hydrogel for $24 \mathrm{~h}$ at $4{ }^{\circ} \mathrm{C}$, followed by polymerization in hydrogel at $37^{\circ} \mathrm{C}$ for $3 \mathrm{~h}$. The brains were cut into $2-$ $\mathrm{mm}$-thick slices. The brain slices were placed in a holder and cleared for $3 \mathrm{~h}$ by electrophoretic tissue clearing with the X-CLARITY Tissue Clearing system (Logos Biosystems, C10001). The cleared brain slices were washed in PBS overnight, and incubated with FITC-conjugated anti-F4/80 antibody (1:100) and Alexa Fluor 647conjugated anti-CD206 antibody (1:100) in PBS for 1 day at $37^{\circ} \mathrm{C}$. After washing with PBS for 1 day, the brain slices were incubated in the X-CLARITY mounting solution (Logos Biosystems, C13101) at $37^{\circ} \mathrm{C}$ for 1 day. The slices were placed on the glass slide for confocal imaging with an SP8 laser microscope (Leica) in the Microscopy Core of the University of Pennsylvania. 3-D images were generated by using the Velocity software.

Immunoblot analysis. Cells and tissues were lysed with a NP-40 buffer containing protease inhibitor cocktail (Roche, 11697498001). Protein (20-50 $\mu \mathrm{g} 0$ was resolved by $4-15 \%$ precast sodium dodecyl-sulfate polyacrylamide gel electrophoresis gel (Bio-Rad). After transfer, PVDF membranes were blotted with anti-GAPDH (1;1,000, Cell Signaling, 5174), anti-PCNA (1:1,000, Origene, TA800875), antimouse arginase-1 (1:1,000, Santa Cruz, sc-18354), anti-human IL-6 (1:1,000, GeneTex, GTX110527), anti-mouse IL-6 (1:1,000, Cell Signaling, 12912), anti- $\alpha-$ tubulin (1:1,000, Cell Signaling, 2144), anti-human CSF-1 (1:1,000, Abcam, ab9693), anti-HIF-1 $\alpha$ (1:1,000, Cayman, 10006421), anti-HIF-2 $\alpha$ (1:1,000, Origene TA309641), anti-PPAR $\left(1: 1,000\right.$, Cell Signaling, 2443), anti-P-Akt-Ser ${ }^{473}$ (1:1,000, Alzforum, AB3132), anti-Akt (1:1,000, Cell Signaling, 4685), anti-P-ERK1/2$\mathrm{Thr}^{202} / \mathrm{Tyr}^{204}$ (1:1,000, Cell Signaling, 4370), anti-ERK1/2 (1:1,000, Cell Signaling 4695), anti-P-mTOR-Ser ${ }^{2448}$ (1:1,000, Cell Signaling, 5536), anti-mTOR (1:1,000, Cell Signaling, 2983), or anti-HSP90 (1:1,000, Cell Signaling, 4874) antibody. Proteins were detected with horseradish peroxidase-conjugated antibodies specific for either rabbit or mouse IgG (Bio-Rad), followed by ECL development (GE Healthcare, RPN2232). Band density was quantified by using NIH Image J program. The uncropped blots were included as in Supplementary Fig. 19.

Flow cytometry. Mouse glioma and normal brain tissues were isolated and subjected to mechanical dissociation with a gentleMACS Dissociator (Miltenyi Biotech) and enzymatic digestion with collagenase II and dispase II. The single-cell suspensions were prepared from mouse tumors and brains, BMDMs cells, and human BMDMs with or without co-culture with ECs. A total of $2 \times 10^{6}$ cells were probed with anti-CD11b (1:100, BioLegend, 101206), anti-CD86 (1:100, BD Pharmingen, 553692), anti-CD206 (1:100, BioLegend,141708), anti-mouse IL-10 (1:100 BioLegend, 505009), anti-mouse IL-12 (1:100, BioLegend, 505203), or anti-human IL-10 (1:100, BioLegend, 506807) antibody with conjugation with different fluorescence dyes, or control IgG. The cells were analyzed with an Accuri C6 flow cytometer (BD Biosciences) by using FlowJo software.

Statistical analysis. Student's $t$ test (unpaired, two-tailed) and log-rank (Mantel-Cox test) analysis were performed by using Prism software for statistical analysis between groups, and $p<0.05$ was considered to represent a statistically significant difference.

Data availability. All data are available within the Article and Supplementary Files, or available from the authors upon request. 
Received: 21 February 2017 Accepted: 16 January 2018

Published online: 08 February 2018

\section{References}

1. Pollard, J. W. Tumour-educated macrophages promote tumour progression and metastasis. Nat. Rev. Cancer 4, 71-78 (2004).

2. Ruffell, B. \& Coussens, L. M. Macrophages and therapeutic resistance in cancer. Cancer Cell 27, 462-472 (2015).

3. Ostuni, R., Kratochvill, F., Murray, P. J. \& Natoli, G. Macrophages and cancer: from mechanisms to therapeutic implications. Trends Immunol. 36, 229-239 (2015).

4. Sica, A. \& Mantovani, A. Macrophage plasticity and polarization: in vivo veritas. J. Clin. Invest. 122, 787-795 (2012).

5. Murray, P. J. \& Wynn, T. A. Protective and pathogenic functions of macrophage subsets. Nat. Rev. Immunol. 11, 723-737 (2011).

6. Stein, M., Keshav, S., Harris, N. \& Gordon, S. Interleukin 4 potently enhances murine macrophage mannose receptor activity: a marker of alternative immunologic macrophage activation. J. Exp. Med. 176, 287-292 (1992).

7. Weber, M. S. et al. Type II monocytes modulate T cell-mediated central nervous system autoimmune disease. Nat. Med. 13, 935-943 (2007).

8. Dunn, G. P. et al. Emerging insights into the molecular and cellular basis of glioblastoma. Genes Dev. 26, 756-784 (2012).

9. Omuro, A. \& DeAngelis, L. M. Glioblastoma and other malignant gliomas: a clinical review. JAMA 310, 1842-1850 (2013).

10. Stupp, R. et al. Radiotherapy plus concomitant and adjuvant temozolomide for glioblastoma. N. Engl. J. Med. 352, 987-996 (2005).

11. Morantz, R. A., Wood, G. W., Foster, M., Clark, M. \& Gollahon, K. Macrophages in experimental and human brain tumors. Part 2: studies of the macrophage content of human brain tumors. J. Neurosurg. 50, 305-311 (1979).

12. Hambardzumyan, D., Gutmann, D. H. \& Kettenmann, H. The role of microglia and macrophages in glioma maintenance and progression. Nat. Neurosci. 19, 20-27 (2016).

13. Pyonteck, S. M. et al. CSF-1R inhibition alters macrophage polarization and blocks glioma progression. Nat. Med. 19, 1264-1272 (2013).

14. Poon, C. C., Sarkar, S., Yong, V. W. \& Kelly, J. J. P. Glioblastoma-associated microglia and macrophages: targets for therapies to improve prognosis. Brain 140, 1548-1560 (2017).

15. Carvalho da Fonseca, A. C. et al. Increased expression of stress inducible protein 1 in glioma-associated microglia/macrophages. J. Neuroimmunol. 274, 71-77 (2014)

16. Coniglio, S. J. et al. Microglial stimulation of glioblastoma invasion involves epidermal growth factor receptor (EGFR) and colony stimulating factor 1 receptor (CSF-1R) signaling. Mol. Med. 18, 519-527 (2012).

17. Wesolowska, A. et al. Microglia-derived TGF-beta as an important regulator of glioblastoma invasion--an inhibition of TGF-beta-dependent effects by shRNA against human TGF-beta type II receptor. Oncogene 27, 918-930 (2008).

18. Vinnakota, K. et al. Toll-like receptor 2 mediates microglia/brain macrophage MT1-MMP expression and glioma expansion. Neuro-Oncology 15, 1457-1468 (2013).

19. Sielska, M. et al. Distinct roles of CSF family cytokines in macrophage infiltration and activation in glioma progression and injury response. J. Pathol. 230, 310-321 (2013).

20. Wang, S. C., Hong, J. H., Hsueh, C. \& Chiang, C. S. Tumor-secreted SDF-1 promotes glioma invasiveness and TAM tropism toward hypoxia in a murine astrocytoma model. Lab Invest. 92, 151-162 (2012).

21. Ku, M. C. et al. GDNF mediates glioblastoma-induced microglia attraction but not astrogliosis. Acta Neuropathol. 125, 609-620 (2013).

22. Martinez, F. O. \& Gordon, S. The M1 and M2 paradigm of macrophage activation: time for reassessment. F1000prime Rep. 6, 13 (2014).

23. Prosniak, M. et al. Glioma grade is associated with the accumulation and activity of cells bearing M2 monocyte markers. Clin. Cancer Res. 19, 3776-3786 (2013).

24. Tugal, D., Liao, X. \& Jain, M. K. Transcriptional control of macrophage polarization. Arterioscler. Thromb. Vasc. Biol. 33, 1135-1144 (2013).

25. Pourcet, B. \& Pineda-Torra, I. Transcriptional regulation of macrophage arginase 1 expression and its role in atherosclerosis. Trends Cardiovasc. Med. 23, 143-152 (2013)

26. Mauer, J. et al. Signaling by IL-6 promotes alternative activation of macrophages to limit endotoxemia and obesity-associated resistance to insulin. Nat. Immunol. 15, 423-430 (2014).

27. Lawrence, T. \& Natoli, G. Transcriptional regulation of macrophage polarization: enabling diversity with identity. Nat. Rev. Immunol. 11, 750-761 (2011).
28. Mazumdar, J., Dondeti, V. \& Simon, M. C. Hypoxia-inducible factors in stem cells and cancer. J. Cell. Mol. Med 13, 4319-4328 (2009).

29. Keith, B. \& Simon, M. C. Hypoxia-inducible factors, stem cells, and cancer. Cell 129, 465-472 (2007).

30. Semenza, G. L. Hypoxia-inducible factors in physiology and medicine. Cell 148, 399-408 (2012).

31. Sumbayev, V. V. LPS-induced Toll-like receptor 4 signalling triggers cross-talk of apoptosis signal-regulating kinase 1 (ASK1) and HIF-1alpha protein. FEBS Lett. 582, 319-326 (2008).

32. Park, J. H. et al. Gastric epithelial reactive oxygen species prevent normoxic degradation of hypoxia-inducible factor-1alpha in gastric cancer cells. Clin. Cancer Res. 9, 433-440 (2003).

33. Selak, M. A. et al. Succinate links TCA cycle dysfunction to oncogenesis by inhibiting HIF-alpha prolyl hydroxylase. Cancer Cell 7, 77-85 (2005).

34. Tannahill, G. M. et al. Succinate is an inflammatory signal that induces IL1beta through HIF-1alpha. Nature 496, 238-242 (2013).

35. Fan, Y. et al. Profilin-1 phosphorylation directs angiocrine expression and glioblastoma progression through HIF-1alpha accumulation. Nat. Cell Biol. 16, 445-456 (2014).

36. Takeda, N. et al. Differential activation and antagonistic function of HIF\{alpha\} isoforms in macrophages are essential for NO homeostasis. Genes Dev. 24, 491-501 (2010).

37. Odegaard, J. I. et al. Macrophage-specific PPARgamma controls alternative activation and improves insulin resistance. Nature 447, 1116-1120 (2007).

38. Bouhlel, M. A. et al. PPARgamma activation primes human monocytes into alternative M2 macrophages with anti-inflammatory properties. Cell Metab. 6 137-143 (2007).

39. Chang, C. Y. et al. Prognostic and clinical implication of IL-6 expression in glioblastoma multiforme. J. Clin. Neurosci. 12, 930-933 (2005).

40. Charles, N. et al. Perivascular nitric oxide activates notch signaling and promotes stem-like character in PDGF-induced glioma cells. Cell Stem Cell 6, 141-152 (2010)

41. Mantovani, A., Sozzani, S., Locati, M., Allavena, P. \& Sica, A. Macrophage polarization: tumor-associated macrophages as a paradigm for polarized M2 mononuclear phagocytes. Trends Immunol. 23, 549-555 (2002).

42. Komohara, Y., Ohnishi, K., Kuratsu, J. \& Takeya, M. Possible involvement of the M2 anti-inflammatory macrophage phenotype in growth of human gliomas. J. Pathol. 216, 15-24 (2008).

43. Gabrusiewicz, K. et al. Glioblastoma-infiltrated innate immune cells resemble M0 macrophage phenotype. JCI Insight 1, pii: e85841 (2016).

44. Beck, B. et al. A vascular niche and a VEGF-Nrp1 loop regulate the initiation and stemness of skin tumours. Nature 478, 399-403 (2011).

45. Bergers, G. \& Hanahan, D. Modes of resistance to anti-angiogenic therapy. Nat. Rev. Cancer 8, 592-603 (2008).

46. Butler, J. M., Kobayashi, H. \& Rafii, S. Instructive role of the vascular niche in promoting tumour growth and tissue repair by angiocrine factors. Nat. Rev. Cancer 10, 138-146 (2010).

47. Calabrese, C. et al. A perivascular niche for brain tumor stem cells. Cancer Cell 11, 69-82 (2007).

48. Carmeliet, P. \& Jain, R. K. Molecular mechanisms and clinical applications of angiogenesis. Nature 473, 298-307 (2011).

49. Ghajar, C. M. et al. The perivascular niche regulates breast tumour dormancy. Nat. Cell Biol. 15, 807-817 (2013).

50. Lu, J. et al. Endothelial cells promote the colorectal cancer stem cell phenotype through a soluble form of jagged-1. Cancer Cell 23, 171-185 (2013).

51. Weis, S. M. \& Cheresh, D. A. Tumor angiogenesis: molecular pathways and therapeutic targets. Nat. Med. 17, 1359-1370 (2011).

52. Cao, Z. et al. Angiocrine factors deployed by tumor vascular niche induce B cell lymphoma invasiveness and chemoresistance. Cancer Cell 25, 350-365 (2014).

53. He, H. et al. Endothelial cells provide an instructive niche for the differentiation and functional polarization of M2-like macrophages. Blood 120, 3152-3162 (2012).

54. Weissenberger, J. et al. IL-6 is required for glioma development in a mouse model. Oncogene 23, 3308-3316 (2004).

55. Kopf, M. et al. Impaired immune and acute-phase responses in interleukin-6deficient mice. Nature 368, 339-342 (1994).

56. Hunter, C. A. \& Jones, S. A. IL-6 as a keystone cytokine in health and disease. Nat. Immunol. 16, 448-457 (2015).

57. Rincon, M. Interleukin-6: from an inflammatory marker to a target for inflammatory diseases. Trends Immunol. 33, 571-577 (2012).

58. Jones, S. A., Scheller, J. \& Rose-John, S. Therapeutic strategies for the clinical blockade of IL-6/gp130 signaling. J. Clin. Invest. 121, 3375-3383 (2011).

59. Fielding, C. A. et al. Interleukin-6 signaling drives fibrosis in unresolved inflammation. Immunity 40, 40-50 (2014).

60. Rincon, M., Anguita, J., Nakamura, T., Fikrig, E. \& Flavell, R. A. Interleukin (IL) 6 directs the differentiation of IL-4-producing CD4+T cells. J. Exp. Med. 185, 461-469 (1997). 
61. Bettelli, E. et al. Reciprocal developmental pathways for the generation of pathogenic effector TH17 and regulatory T cells. Nature 441, 235-238 (2006).

62. Ricote, M., Li, A. C., Willson, T. M., Kelly, C. J. \& Glass, C. K. The peroxisome proliferator-activated receptor-gamma is a negative regulator of macrophage activation. Nature 391, 79-82 (1998).

63. Szanto, A. et al. STAT6 transcription factor is a facilitator of the nuclear receptor PPARgamma-regulated gene expression in macrophages and dendritic cells. Immunity 33, 699-712 (2010).

64. Liao, X. et al. Kruppel-like factor 4 regulates macrophage polarization. J. Clin. Invest. 121, 2736-2749 (2011).

65. Hamidian, A., von Stedingk, K., Munksgaard Thoren, M., Mohlin, S. \& Pahlman, S. Differential regulation of HIF-1alpha and HIF-2alpha in neuroblastoma: estrogen-related receptor alpha (ERRalpha) regulates HIF2A transcription and correlates to poor outcome. Biochem. Biophys. Res. Commun. 461, 560-567 (2015).

66. Huang, M. et al. c-Met-mediated endothelial plasticity drives aberrant vascularization and chemoresistance in glioblastoma. J. Clin. Invest. 126, 1801-1814 (2016).

67. Wang, Y. et al. Ephrin-B2 controls VEGF-induced angiogenesis and lymphangiogenesis. Nature 465, 483-486 (2010).

68. Quintana, A. et al. Astrocyte-specific deficiency of interleukin-6 and its receptor reveal specific roles in survival, body weight and behavior. Brain Behav. Immun. 27, 162-173 (2013).

69. Molnarfi, N. et al. MHC class II-dependent B cell APC function is required for induction of CNS autoimmunity independent of myelin-specific antibodies. J. Exp. Med. 210, 2921-2937 (2013).

70. Nakazawa, M. S. et al. Epigenetic re-expression of HIF-2alpha suppresses soft tissue sarcoma growth. Nat. Commun. 7, 10539 (2016).

\section{Acknowledgements}

We are grateful to Eric Holland for providing RCAS-PDGF GBM model, to Celeste Simon for providing reagents and comments for HIF-related research, and to Laura Johnson and Andrea Facciabene for helpful discussions. This work was supported in part by National Institutes of Health grants R00HL103792 and R01NS094533 (to Y.F.) and R01CA190415 (to L.Z.), American Association for Cancer Research Judah Folkman Award (to Y.F.), $B^{\star}$ Cured Foundation Brain Cancer Investigator Award (to. Y.F.), and National Brain Tumor Society Sharpe Award (to Y.F.). J.H. is supported by SAF201123272 and SAF2014-56546-R.

\section{Author contributions}

Q.W. designed, performed, and analyzed experiments, produced figures, and wrote the manuscript. Z.H. and T.L. helped tumor induction in mice and contributed to flow cytometry analysis. M.H. contributed to cytokine array analysis and 3-D confocal imaging. Y.W. generated IL-6R $\alpha$ shRNAs. H.X. helped multiplex transcriptional activity assay. P.M. contributed to tumor section preparation and immunofluorescence analysis. S.S.Z. and J.H. contributed to the generation of endothelial-specific IL-6 knockout mice H.D. and Y.M. contributed to tumor section preparation. D.M.O.R. and N.D. contributed to preparation of single-cell suspension of human glioblastoma tumors. L.Z., Z.Z., S.B., and Y.G. provided suggestions for experimental design. Y.F. designed and supervised experiments and wrote the manuscript. All authors commented on the manuscript.

\section{Additional information}

Supplementary Information accompanies this paper at https://doi.org/10.1038/s41467018-03050-0.

Competing interests: The authors declare no competing financial interests.

Reprints and permission information is available online at http://npg.nature.com/ reprintsandpermissions/

Publisher's note: Springer Nature remains neutral with regard to jurisdictional claims in published maps and institutional affiliations. (c) Open Access This article is licensed under a Creative Commons
Attribution 4.0 International License, which permits use, sharing, adaptation, distribution and reproduction in any medium or format, as long as you give appropriate credit to the original author(s) and the source, provide a link to the Creative Commons license, and indicate if changes were made. The images or other third party material in this article are included in the article's Creative Commons license, unless indicated otherwise in a credit line to the material. If material is not included in the article's Creative Commons license and your intended use is not permitted by statutory regulation or exceeds the permitted use, you will need to obtain permission directly from the copyright holder. To view a copy of this license, visit http://creativecommons.org/ licenses/by/4.0/.

(C) The Author(s) 2018 